\title{
Theory and Computation of Non-RRKM Lifetime Distributions and Rates in Chemical Systems with Three or More Degrees of Freedom
}

\author{
Frederic Gabern, Wang S. Koon, Jerrold E. Marsden \\ Control and Dynamical Systems \\ California Institute of Technology, MC 107-81 \\ Pasadena, CA 91125 \\ Shane D. Ross \\ Department of Aerospace and Mechanical Engineering \\ University of Southern California, RRB 217 \\ Los Angeles, CA 90089-1191
}

February 20, 2005

\begin{abstract}
The computation, starting from basic principles, of chemical reaction rates in realistic systems (with three or more degrees of freedom) has been a longstanding goal of the chemistry community. Our current work, which merges tube dynamics with Monte Carlo methods provides some key theoretical and computational tools for achieving this goal. We use basic tools of dynamical systems theory, merging the ideas of Koon et al. [Chaos 10, 427 (2000)] and De Leon et al. [J. Chem. Phys. 94, 8310 (1991)], particularly the use of invariant manifold tubes that mediate the reaction, into the start of a comprehensive theory of lifetime distributions and rates of chemical reactions and scattering phenomena, even in systems that exhibit non-statistical behavior. Previously, the main problem with the application of tube dynamics has been with the analytical evaluation of volumes in phase spaces of arbitrary dimension. The present work overcomes this hurdle with some new ideas and implements them numerically. Specifically, an efficient algorithm that uses tube dynamics to provide the initial bounding box for a Monte Carlo volume determination is used. The combination of a fine scale method for understanding the phase space structure (invariant manifold theory) with statistical methods for practical computations (Monte Carlo) is the main novel contribution of this paper. The methodology, applied here to a three degree of freedom model problem, is not restricted by dimension, and is useful for higher degree of freedom systems as well.
\end{abstract}

*Present address: Departament de Matemàtica Aplicada i Anàlisi, Universitat de Barcelona, Gran Via 585, 08007 Barcelona, Spain. 


\section{Contents}

1 Introduction 2

2 General Methodology 3

2.1 Phase Space Structure near the Saddle . . . . . . . . . . . . . . . . . . . 4

2.2 Global Transport and Poincaré Cuts . . . . . . . . . . . . . . . . . . 9 9

2.3 Numerical Computation of the Lifetime Distribution Spectrum . . . . . . . . . 11

3 Application to Rydberg Atom Formation and Ionization 13

3.1 The Hamiltonian Model . . . . . . . . . . . . . . . . . . . . . . . . . . . 13

3.2 NHIM and the Stable and Unstable Tubes . . . . . . . . . . . . . . . . . . . . 14

3.3 Intersection Volumes, Lifetime Distributions, and Rates . . . . . . . . . . . 18

4 Discussion $\quad 20$

A Effective Computation of NHIM and its Stable and Unstable Manifolds 21

References $\quad 24$

\section{Introduction}

The goal of this paper is to begin a program of computation of chemical reaction rates in realistic models of molecular systems, with three or more degrees of freedom (dof). Rates computed using standard statistical methods, such as Rice-Ramsperger-Kassel-Marcus (RRKM) [14] theory can be several orders of magnitude off of experimental values [5]. Despite its shortcomings, RRKM theory has been a workhorse of the chemistry community for decades. RRKM theory, also known as transition state theory (TST) [45], is based on the identification of a transition state (TS) between large regions of phase space that correspond to either "reactants" or "products" and it assumes that the phase space in each region is structureless [33]. However, it is now well known that while this basic assumption is true in some limited circumstances, in general these regions (often defined by potential wells) are by no means structureless [23].

De Leon et al. [6, 7] attempted to extend the local picture near the TS in two degree of freedom systems to a more global one and developed reaction island theory using cylindrical manifolds [38] (now known as tubes [41]). Berry and collaborators (see for instance [20]) studied the local regular behavior near the saddle regions by means of Kolmogorov entropies. Marcus [32] suggested that these regularities were due to the existence of some invariants near the TS. Komatsuzaki and Berry [26, 27, 28] made further progress by using dynamical perturbative methods to study the transition near the saddle region. Uzer et al. [46], by using a general dynamical systems framework, studied the local geometric structures of rank-one saddles that regulate reactions in systems with three or more dof. Recently, in Waalkens et al. [47], homoclinic and heteroclinic orbits in a tri-atomic molecule have been computed. But a comprehensive theory of chemical reactions and efficient computational tools for reaction rate calculations in three or more dof systems which takes into consideration phase space structures still needs to be developed. We are now poised to make progress toward more accurate theories due to (i) advances in dynamical systems theory, especially tube dynamics, and (ii) increased computing power. We initiate progress in this direction in the present paper.

The current work, which merges tube dynamics with Monte Carlo methods, provides some enabling theoretical and computational tools needed for accurate rate calculations. In this paper, we present a general methodology that uses basic tools of dynamical systems theory, merging the ideas of $[29,18]$ and De Leon et al. (see, e.g., $[6,7]$ ). In particular, we use invariant 
manifold tubes mediating the dynamical process of reaction as the start of a comprehensive theory of lifetime distributions and rates of chemical reactions and scattering phenomena. The standard RRKM assumption of an unstructured phase space fails to account for the dynamics of systems exhibiting significant non-statistical behavior. We overcome this difficulty by taking into consideration the homoclinic and heteroclinic intersection structure of tubes in the phase space. Furthermore, by working in the phase space as opposed to configuration space, we overcome the recrossing problem, i.e., the recrossing of the transition state as projected onto configuration space, which if uncorrected leads to inaccurate rate computations.

Previously, the main problem with the application of tube dynamics has been with the analytical evaluation of volumes in phase spaces of arbitrary dimension $[7,46]$. The present work overcomes this hurdle by using an efficient algorithm that uses tube dynamics to provide the initial bounding box for a Monte Carlo volume determination. The main contribution of the paper is the combination of an accurate method for computing and understanding invariant manifolds in the problem and hence the phase space structure together with statistical Monte Carlo methods for practical computations.

We show the practical applicability of the methodology in a model problem in which the hypotheses of TST do not hold: namely, the full-scattering of electrons in Rydberg atoms in the presence of external crossed electric and magnetic fields. We use a variety of methods and software that have been developed in the last several years for tube dynamics [24, 29, 18, 41] to better understand the transport between different regions (or realms) of phase space. The numerical results obtained are a demonstration of accurate lifetime distribution and rate calculations which overcome the difficulties that have plagued the standard statistical methods.

The paper is organized as follows: In Section 2, we describe the global geometric structure of the phase space for reactions between two regions connected via a rank-one saddle point. We also introduce the general methodology for the computation of scattering rates and lifetime distributions. The computational tool employed to produce these detailed structures is based on normal form techniques and is reviewed in the appendix for the convenience of the reader. In Section 3, we apply the general methodology of Section 2 to the scattering problem of Rydbergtype atoms in crossed electric and magnetic fields. Finally, in Section 4, we make several additional remarks and point out some possible directions for future work.

\section{General Methodology}

Many chemical reactions and some scattering phenomena proceed through energetic barriers. In general, such situations are well described in phase space where the energy-fixed hyper-surface determines different regions that are connected by the energy barriers, specifically by structures related to rank-one saddles associated with the barriers. To make the discussion that follows as simple as possible, we consider a two state system where one state is bound and the other is unbound. We will refer to this problem as a scattering problem for purposes of the present discussion.

The simplest case is shown in Figure 1 where a bound region (zone on the left of the bottleneck) is next to an unbound region (unbound zone on the right of the bottleneck), and the bottleneck takes place precisely at the rank-one saddle equilibrium point. More concretely, this figure shows a planar projection of the Hill region for the model problem used in this paper, namely a Rydberg atom in crossed electric and magnetic fields. Recall that the Hill region is the projection of the energy surface onto the position space. In the figure, the white zone corresponds to the portion of the position space where the motion is possible for the given level of energy. 


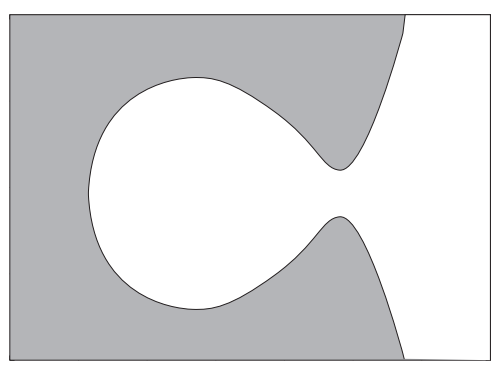

(a)

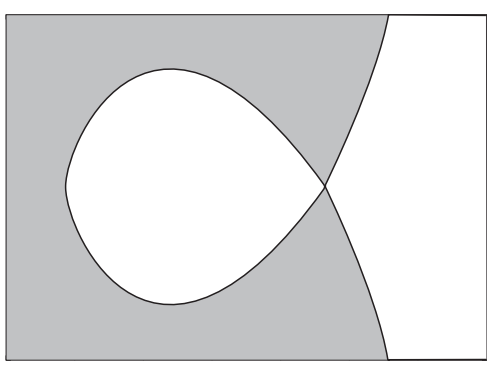

(b)

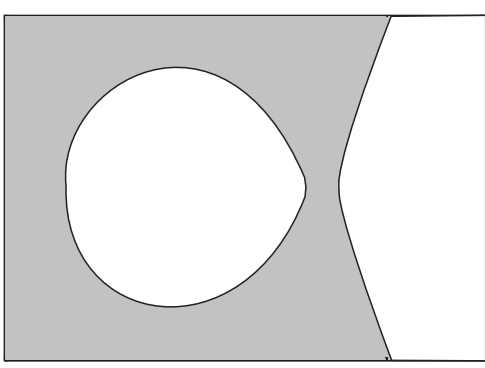

(c)

Figure 1: Planar projections of the actual Hill region for the Rydberg atom in crossed electric and magnetic fields. The three possible cases are shown: Connected (a) and unconnected (c) bound and unbound states separated by a bottleneck related to a rank-one saddle. The connectivity depends on the energy level. Case (b) corresponds to the critical case.

\subsection{Phase Space Structure near the Saddle}

Studying the linearization of the dynamics near the saddle equilibrium point is of course an essential ingredient for understanding the more complete nonlinear dynamics [29]. In fact, it can be shown that for a value of energy constant just above that of the saddle, the nonlinear dynamics in the equilibrium region is qualitatively the same as the linearized picture that we will describe below. For details, see the appendix at the end of this paper as well as other references [25, 51]. However, since this geometric insight will be used later to guide our numerical algorithms for effectively computing non-statistical lifetime distributions for scattering problems, a brief review of the linearized picture will be provided below for the benefit of the readers.

The Linear Dynamics Near the Saddle. Assume we are dealing with a Hamiltonian system for which preliminary linear transformations have been performed (essentially, a translation to put the saddle at the origin and a linear change that uses the eigenvectors of the linear system as the new basis) so that the Hamiltonian function for the linear system near the saddle has the following quadratic (normal) form:

$$
H_{2}\left(q_{1}, p_{1}, \ldots, q_{n}, p_{n}\right)=\lambda q_{1} p_{1}+\sum_{k=2}^{n} \frac{\omega_{k}}{2}\left(q_{k}^{2}+p_{k}^{2}\right),
$$

where $n$ is the number of degrees of freedom, $\lambda$ is the real eigenvalue corresponding to the hyperbolic direction spanned by $\left(q_{1}, p_{1}\right), \omega_{k}$ are the bath mode frequencies; that is, the frequencies associated to the variables $\left(q_{2}, p_{2}, q_{3}, p_{3}, \ldots, q_{n}, p_{n}\right)$. See the appendix for details.

By fixing a positive $h \in \mathbb{R}$ and $c \in \mathbb{R}$, we define a region $\mathcal{R} \subset \mathbb{R}^{2 n}$ in phase space by the condition

$$
H_{2}\left(q_{1}, p_{1}, \ldots, q_{n}, p_{n}\right)=h, \quad \text { and }\left|p_{1}-q_{1}\right| \leq c .
$$

It can be seen that $\mathcal{R}$ is homeomorphic to the product of a $(2 n-2)$-sphere and an interval $I$, that is, $\mathcal{R} \cong S^{2 n-2} \times I$; namely, for each fixed value of $\left(p_{1}-q_{1}\right)$ in the interval $I=[-c, c]$, we see that the equation $H_{2}\left(q_{1}, p_{1}, \ldots, q_{n}, p_{n}\right)=h$ determines a $(2 n-2)$-sphere

$$
\frac{\lambda}{4}\left(q_{1}+p_{1}\right)^{2}+\sum_{k=2}^{n} \frac{\omega_{k}}{2}\left(q_{k}^{2}+p_{k}^{2}\right)=h+\frac{\lambda}{4}\left(p_{1}-q_{1}\right)^{2} .
$$

The bounding $(2 n-2)$-sphere of $\mathcal{R}$ for which $p_{1}-q_{1}=-c$ will be called $n_{1}$, and that where $p_{1}-q_{1}=c, n_{2}$ (see Figure 2 ). We call the set of points on each bounding $(2 n-2)$-sphere where 


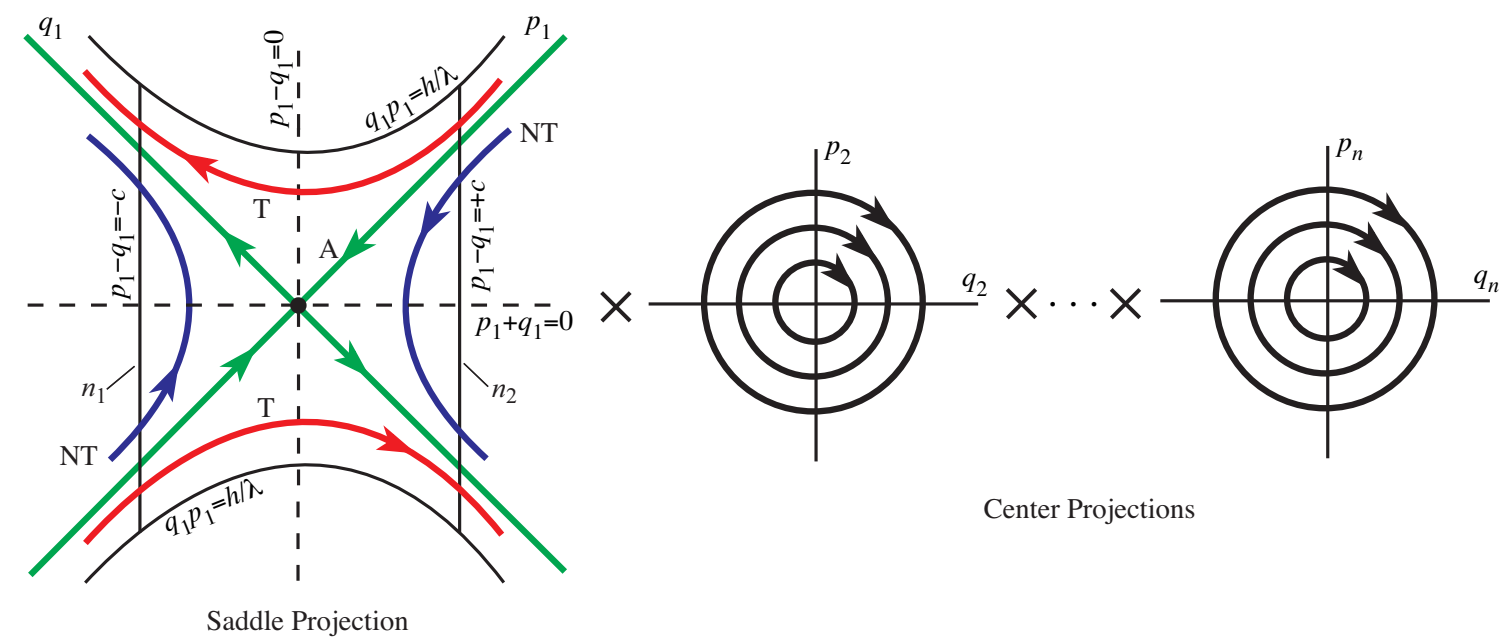

Figure 2: The flow in the equilibrium region has the form saddle $\times$ center $\times \cdots \times$ center. On the left is shown the projection onto the $\left(p_{1}, q_{1}\right)$-plane (note, axes tilted $45^{\circ}$ ). Shown are the NHIM (black dot at the center), the asymptotic orbits (labeled A), two transit orbits (T) and two non-transit orbits (NT).

$q_{1}+p_{1}=0$ the equator, and the sets where $q_{1}+p_{1}>0$ or $q_{1}+p_{1}<0$ will be called the north and south hemispheres, respectively. Notice the $(2 n-2)$-sphere at the middle of the equilibrium region where $p_{1}-q_{1}=0$. This sphere, which is defined as follows

$$
\mathcal{N}_{h}^{2 n-2}=\left\{(q, p) \mid \lambda p_{1}^{2}+\sum_{k=2}^{n} \frac{\omega_{k}}{2}\left(q_{k}^{2}+p_{k}^{2}\right)=h\right\},
$$

corresponds to the transition state in the chemical literature and plays an important role in chemical reaction dynamics, as we will see later.

To analyze the flow in $\mathcal{R}$, one considers the projections on the $\left(q_{1}, p_{1}\right)$-plane and $\left(q_{2}, p_{2}\right) \times$ $\cdots \times\left(q_{n}, p_{n}\right)$-space, respectively. In the first case we see the standard picture of an unstable critical point, and in the second, of a center consisting of $(n-1)$ uncoupled harmonic oscillators. Figure 2 schematically illustrates the flow. Notice that $\mathcal{R}$ itself projects to a set bounded on two sides by the hyperbola $q_{1} p_{1}=h / \lambda$ (corresponding to $q_{2}^{2}+p_{2}^{2}=\cdots=q_{n}^{2}+p_{n}^{2}=0$, see (1)) and on two other sides by the line segments $p_{1}-q_{1}= \pm c$, which correspond to the bounding $(2 n-2)$-spheres.

Since $q_{1} p_{1}$ is an integral of the (linearized) equations in $\mathcal{R}$, the projections of orbits in the $\left(q_{1}, p_{1}\right)$-plane move on the branches of the corresponding hyperbolas $q_{1} p_{1}=$ constant, except in the case $q_{1} p_{1}=0$, in which case $q_{1}=0$ or $p_{1}=0$. If $q_{1} p_{1}>0$, the branches connect the bounding line segments $p_{1}-q_{1}= \pm c$. If $q_{1} p_{1}<0$, they have both end points on the same segment.

To interpret Figure 2 as a flow in $\mathcal{R}$, notice that each point in the $\left(q_{1}, p_{1}\right)$-plane projection corresponds to a $(2 n-3)$-sphere $S^{2 n-3}$ in $\mathcal{R}$ given by

$$
\sum_{k=2}^{n} \frac{\omega_{k}}{2}\left(q_{k}^{2}+p_{k}^{2}\right)=h-\lambda q_{1} p_{1}
$$

Of course, for points on the bounding hyperbolic segments $\left(q_{1} p_{1}=h / \lambda\right)$, the $(2 n-3)$-sphere collapses to a point. Thus, the segments of the lines $p_{1}-q_{1}= \pm c$ in the projection correspond 
to the $(2 n-2)$-spheres bounding $\mathcal{R}$. This is because each corresponds to a $(2 n-3)$-sphere crossed with an interval where the two end $(2 n-3)$-spheres are pinched to a point.

The following objects are relevant for understanding transport through the saddle:

1. The point $q_{1}=p_{1}=0$ corresponds to an invariant $(2 n-3)$-sphere $S_{h}^{2 n-3}$ of periodic and quasi-periodic orbits in $\mathcal{R}$. This $(2 n-3)$-sphere is given by

$$
\sum_{k=2}^{n} \frac{\omega_{k}}{2}\left(q_{k}^{2}+p_{k}^{2}\right)=h, \quad q_{1}=p_{1}=0 .
$$

This is known in the literature [50] as a normally hyperbolic invariant manifold (NHIM). Roughly, this means that the stretching and contraction rates under the linearized dynamics transverse to the $(2 n-3)$-sphere dominate those tangent to the $(2 n-3)$-sphere. This is clear since the dynamics normal to the $(2 n-3)$-sphere are described by the exponential contraction and expansion of the saddle point dynamics. The $(2 n-3)$-sphere acts as a "big saddle point". See the black dot at the center of the $\left(q_{1}, p_{1}\right)$-plane on the left side of Figure 2. Note that the NHIM is the equator of the transition state $\mathcal{N}_{h}^{2 n-2}$ and divides it into north and south hemispheres.

2. The four half open segments on the axes, $q_{1} p_{1}=0$, correspond to four high-dimensional cylinders of orbits asymptotic to this invariant $(2 n-3)$-sphere $S_{h}^{2 n-3}$ either as time increases $\left(p_{1}=0\right)$ or as time decreases $\left(q_{1}=0\right)$. These are called asymptotic orbits and they form the stable and the unstable manifolds of $S_{h}^{2 n-3}$. The stable manifolds, $W_{ \pm}^{s}\left(S_{h}^{2 n-3}\right)$, are given by

$$
\sum_{k=2}^{n} \frac{\omega_{k}}{2}\left(q_{k}^{2}+p_{k}^{2}\right)=h, \quad q_{1}=0 .
$$

$W_{+}^{s}\left(S_{h}^{2 n-3}\right.$ ) (with $p_{1}>0$ ) is the branch going from right to left (from the unbound state to the saddle region) and $W_{-}^{s}\left(S_{h}^{2 n-3}\right)$ (with $p_{1}<0$ ) is the branch going from left to right (from the bound state to the saddle region). The unstable manifolds, $W_{ \pm}^{u}\left(S_{h}^{2 n-3}\right)$, are given by

$$
\sum_{k=2}^{n} \frac{\omega_{k}}{2}\left(q_{k}^{2}+p_{k}^{2}\right)=h, \quad p_{1}=0 .
$$

$W_{+}^{u}\left(S_{h}^{2 n-3}\right.$ ) (with $q_{1}>0$ ) is the branch going from right to left (from the saddle region to the bound state) and $W_{-}^{u}\left(S_{h}^{2 n-3}\right)$ (with $q_{1}<0$ ) is the branch going from left to right (from the saddle region to the unbound state). See the four orbits labeled A in Figure 2. There are four cylinders of orbits asymptotic to the invariant $(2 n-3)$-sphere $S_{h}^{2 n-3}$. They form the stable and unstable manifolds to the invariant $(2 n-3)$-sphere $S_{h}^{2 n-3}$. Topologically, both invariant manifolds look like $(2 n-2)$-dimensional "tubes" $\left(S^{2 n-3} \times \mathbb{R}\right)$ inside a $(2 n-1)$-dimensional energy manifold. See Figure $4(\mathrm{a})$ for examples of these structures.

3. The hyperbolic segments determined by $q_{1} p_{1}=$ constant $>0$ correspond to two cylinders of orbits that cross $\mathcal{R}$ from one bounding $(2 n-2)$-sphere to the other, meeting both in the same hemisphere; the northern hemisphere if they go from $p_{1}-q_{1}=+c$ to $p_{1}-q_{1}=-c$, and the southern hemisphere in the other case. Since these orbits transit from one region to another passing through the $(2 n-2)$-sphere $\mathcal{N}_{h}^{2 n-2}$ which is the transition state in the linearized system, we call them transit orbits. See the two orbits labeled T in Figure 2.

4. Finally the hyperbolic segments determined by $q_{1} p_{1}=$ constant $<0$ correspond to two cylinders of orbits in $\mathcal{R}$ each of which runs from one hemisphere to the other hemisphere on the same bounding 4-sphere. Thus if $q_{1}>0$, the 4-sphere is $n_{1}\left(p_{1}-q_{1}=-c\right)$ and orbits 
run from the southern hemisphere $\left(q_{1}+p_{1}<0\right)$ to the northern hemisphere $\left(q_{1}+p_{1}>0\right)$ while the converse holds if $q_{1}<0$, where the 4 -sphere is $n_{2}$. Since these orbits return to the same region and they do not pass through the transition state $\mathcal{N}_{h}^{2 n-2}$, we call them non-transit orbits. See the two orbits labeled NT of Figure 2.

5. The key observation here is that the asymptotic orbits form $(2 n-2)$-dimensional stable and unstable manifold tubes $\left(S^{2 n-3} \times \mathbb{R}\right)$ to the invariant $(2 n-3)$-sphere $S_{h}^{2 n-3}$ in a $(2 n-1)$ dimensional energy surface and thus, they separate two distinct types of motion: transit orbits and non-transit orbits. The transit orbits, passing from one region to another, are those inside the $(2 n-2)$-dimensional manifold tube. The non-transit orbits, which bounce back to their region of origin, are those outside the tube.

Remark on History and Cross-Fertilization. It is interesting to note that some of the same phase space structures and techniques described above that are useful in the chemistry context, were first used in a celestial mechanics setting by Conley and McGehee in the 1960s $[3,4,34]$. Conversely, techniques from chemistry have been used in celestial problems, as was done in [23]. Due do the $N$-body nature and Hamiltonian underpinnings of both fields, we expect this type of fruitful cross-fertilization to continue.

Nonlinear Dynamics and Separatrices. For a value of the energy just above that of the saddle, the nonlinear dynamics in the equilibrium region $\mathcal{R}$ is qualitatively the same as the linearized picture that we have shown above $[36,51]$.

For example, the NHIM for the nonlinear system which corresponds to the $(2 n-3)$-sphere in equation (3) for the linearized system is given by

$$
\mathcal{M}_{h}^{2 n-3}=\left\{(q, p) \mid \sum_{k=2}^{n} \frac{\omega_{k}}{2}\left(q_{k}^{2}+p_{k}^{2}\right)+f\left(q_{2}, p_{2}, \ldots, q_{n}, p_{n}\right)=h, \quad q_{1}=p_{1}=0\right\}
$$

where $f$ is at least of third order. Here, $\left(q_{2}, p_{2}, \ldots, q_{n}, p_{n}\right)$ are normal form coordinates and are related to the linearized coordinates via a near-identity transformation.

In a small neighborhood of the equilibrium point, since the higher order terms in $f$ are much smaller than the second order terms, the $(2 n-3)$-sphere for the linear problem becomes a deformed sphere for the nonlinear problem. Moreover, since NHIMs persist under perturbation, this deformed sphere $\mathcal{M}_{h}^{2 n-3}$ still has stable and unstable manifolds that are given by

$$
\begin{aligned}
& W_{ \pm}^{s}\left(\mathcal{M}_{h}^{2 n-3}\right)=\left\{(q, p) \mid \sum_{k=2}^{n} \frac{\omega_{k}}{2}\left(q_{k}^{2}+p_{k}^{2}\right)+f\left(q_{2}, p_{2}, \ldots, q_{n}, p_{n}\right)=h, \quad q_{1}=0\right\} \\
& W_{ \pm}^{u}\left(\mathcal{M}_{h}^{2 n-3}\right)=\left\{(q, p) \mid \sum_{k=2}^{n} \frac{\omega_{k}}{2}\left(q_{k}^{2}+p_{k}^{2}\right)+f\left(q_{2}, p_{2}, \ldots, q_{n}, p_{n}\right)=h, \quad p_{1}=0\right\} .
\end{aligned}
$$

Notice the similarity between the formulas above and those for the linearized problem given by equation (4) and (5). The same observation also holds for the transition state: in the nonlinear system, it is a deformed $(2 n-2)$-sphere.

See the appendix at the end of the paper as well as other references $[42,19,25,51]$ for details on the semi-analytical approximation of these objects. This geometric insight will be used below to guide our numerical algorithms for computing reaction and scattering rates.

Remarks on Recrossing and Lingering Motions. From the local analysis above, it should be clear that for a value of energy constant just above that of the saddle, transit trajectories will not locally recross the transition state in the phase space. However, they may 
linger in the saddle region for a certain amount of time; the lingering time of a trajectory depends inversely on its distance from the invariant manifold tube. Clearly, if the trajectory is on the invariant manifold, it will asymptotically wind on to the NHIM and stay in the saddle region for ever. On the other hand, if the trajectory is in the middle of the tube, it will passes the saddle region the fastest. In Figure 3(a), we shown a schematic picture of the cross section of the tube. Trajectories crossing this section in the center are the fastest. The time profile shows a logarithmic growth (see Figure 3(b,c)) as the trajectories approach the boundary of the tube. Figures $3(\mathrm{~b}, \mathrm{c})$ correspond to actual computations in the model problem, a Rydberg atom in crossed electric and magnetic fields.

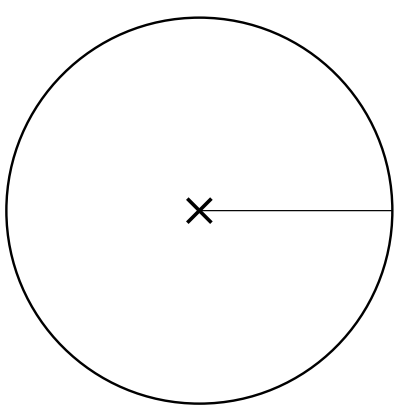

(a)

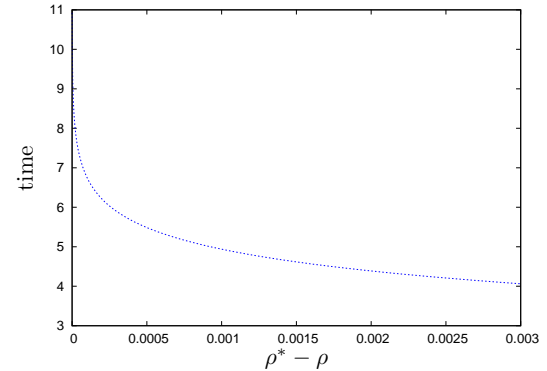

(b)

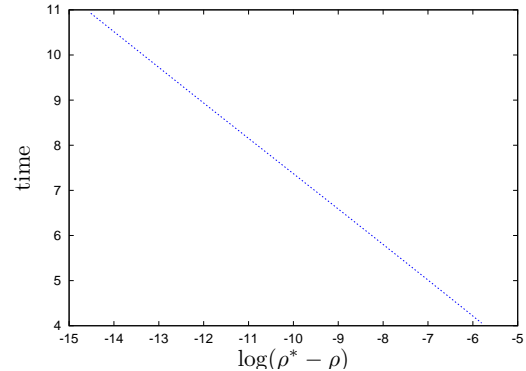

(c)

Figure 3: (a) Schematic cross section of the tube near the Transition State. The center of the tube corresponds to the trajectory that passes the TS the fastest. The boundary of the tube is formed by asymptotic orbits to the NHIM. As we approach this boundary, the time needed to cross the TS approaches infinity. This is shown in (b), where we plot the time taken by different trajectories from a particular Poincaré section to the TS. This time is logarithmic with resepct to the distance to the border of the tube. This is shown in (c), where we plot the log version of (b). Figures (b) and (c) correspond to actual computations for the model problem, the Rydberg atom in crossed electric and magnetic fields. We choose a random mesh of points inside the tube on a Poincaré section in the region corresponding to bounded orbits and compute the time they need to cross the TS depending on the distance to its border (NHIM). See the text for more details.

The transit time $T$ through a saddle can also be analytically estimated by the following formula:

$$
T=\frac{1}{\lambda}\left(\ln \frac{\lambda\left(p_{1}(0)\right)^{2}}{\left(\rho^{*}-\rho\right)}\right)=\frac{1}{\lambda}\left(\ln \lambda\left(p_{1}(0)\right)^{2}-\ln \left(\rho^{*}-\rho\right)\right)
$$

where the last term determines the order of the required transit time (see [2]). Here, $\rho$ is the "bath" variable defined as follow

$$
\rho:=\sum_{k=2}^{n} \frac{\omega_{k}}{2}\left(q_{k}^{2}+p_{k}^{2}\right)=h-\lambda q_{1} p_{1} .
$$

And $\rho^{*}=h$ is the value of the bath variable corresponding to asymptotic orbits $q_{1} p_{1}=0$ which form the boundary of the tube. Clearly, the transit time approaches infinity as the flow approaches the boundary $\left(\rho \rightarrow \rho^{*}\right)$, since orbits on the boundary of the tube are asymptotic to the NHIM.

The proof of the formula (7) is quite straightforward. Take the case of a transit trajectory starting from the northern hemisphere of $n_{2}$ and ending at the northern hemisphere of $n_{1}$. The initial condition of this trajectory $\left\{q_{1}(0), p_{1}(0)\right\}$ are both positive and

$$
p_{1}(0)-q_{1}(0)=+c .
$$


Similarly, if $T$ is the time required to go from $n_{2}$ to $n_{1}$, then $p_{1}(T)=p_{1}(0) e^{\lambda T}$ and $q_{1}(T)=$ $q_{1}(0) e^{-\lambda T}$ on $n_{1}$ where

$$
p_{1}(T)-q_{1}(T)=p_{1}(0) e^{-\lambda T}-q_{1}(0) e^{\lambda T}=-c .
$$

Eliminating $c$ from the two above equations and solving for $T$, we obtain

$$
T=\frac{1}{\lambda} \ln \frac{p_{1}(0)}{q_{1}(0)} .
$$

Moreover, the energy integral gives

$$
p_{1}(0) q_{1}(0)=\frac{1}{\lambda}\left(h-\sum_{k=2}^{n} \frac{\omega_{k}}{2}\left(q_{k}^{2}+p_{k}^{2}\right)\right)=\frac{1}{\lambda}\left(\rho^{*}-\rho\right) .
$$

After a few steps of algebraic manipulations, we obtain the formula (7).

\subsection{Global Transport and Poincaré Cuts}

We have just seen that the stable and unstable manifolds of the NHIM act as separatrices in phase space. They are the geometric structures that completely control the transport between the bound and unbound regions and, consequently, the chemical reaction rates and scattering lifetime distributions.

In this section, we study in detail the reaction mechanism and develop a technique for the computation of the corresponding rates. As is usual in this kind of computation, we use carefully chosen $(2 n-2)$-dimensional Poincaré sections $\Sigma_{h}$ in the $(2 n-1)$-dimensional energy surface to simplify the problem.

We proceed as follows. The unbound and bound regions are labeled in Figure 4(a) as $R_{U}$ and $R_{B}$, respectively. Any reaction trajectory going from an unbound state to a bound state must initially be in the interior of the stable tube $W_{+}^{s}$, and continues in the interior of the unstable $W_{+}^{u}$ tube. These two + branches, $W_{+}^{s} \cup W_{+}^{u}$, constitute the capture reaction path from the unbound to bound state. This reaction path will first pierce the Poincaré section in the entrance or first Poincaré cut $\bar{C}_{+}^{1}$ (the first forward intersection of the interior of the unstable tube $W_{+}^{u}$ with the Poincaré section). Similarly, the two - branches, $W_{-}^{s} \cup W_{-}^{u}$, constitute the escape reaction path, and any reaction trajectory from the bound state to the unbound state has to pass through the exit or first Poincaré cut $\bar{C}_{-}^{1}$ (the first backward intersection of the interior of the stable tube $W_{-}^{s}$ with the Poincaré section) of this reaction path, just before reaction takes place. Figures $4(\mathrm{a}, \mathrm{b})$ show the actual computations of these structures for our model problem, the Rydberg atom in crossed electric and magnetic fields. In Figure 4(a), we plot the projections onto the $x y$ plane of the stable and unstable manifolds of the NHIM and in Figure 4(b), examples of $x p_{x}$ Poincaré cuts are shown.

After defining the Poincaré return map $f$ on $\Sigma_{h}$, we can denote the images of the entrance $\bar{C}_{+}^{1}$ as

$$
\bar{C}_{+}^{m}=f^{m-1}\left(\bar{C}_{+}^{1}\right),
$$

which is the $m$-th forward intersection of the capture reaction path with the Poincare section.

Similarly, we can denote the pre-images of the exit $\bar{C}_{-}^{1}$ as

$$
\bar{C}_{-}^{k}=f^{-(k-1)}\left(\bar{C}_{-}^{1}\right),
$$

which is the $k$-th backward intersection of the escape reaction path with the Poincare section.

The intersection of the images of the entrance and the pre-images of the exit under the Poincaré return map

$$
f^{m-1}\left(\bar{C}_{+}^{1}\right) \cap f^{-(k-1)}\left(\bar{C}_{-}^{1}\right)
$$




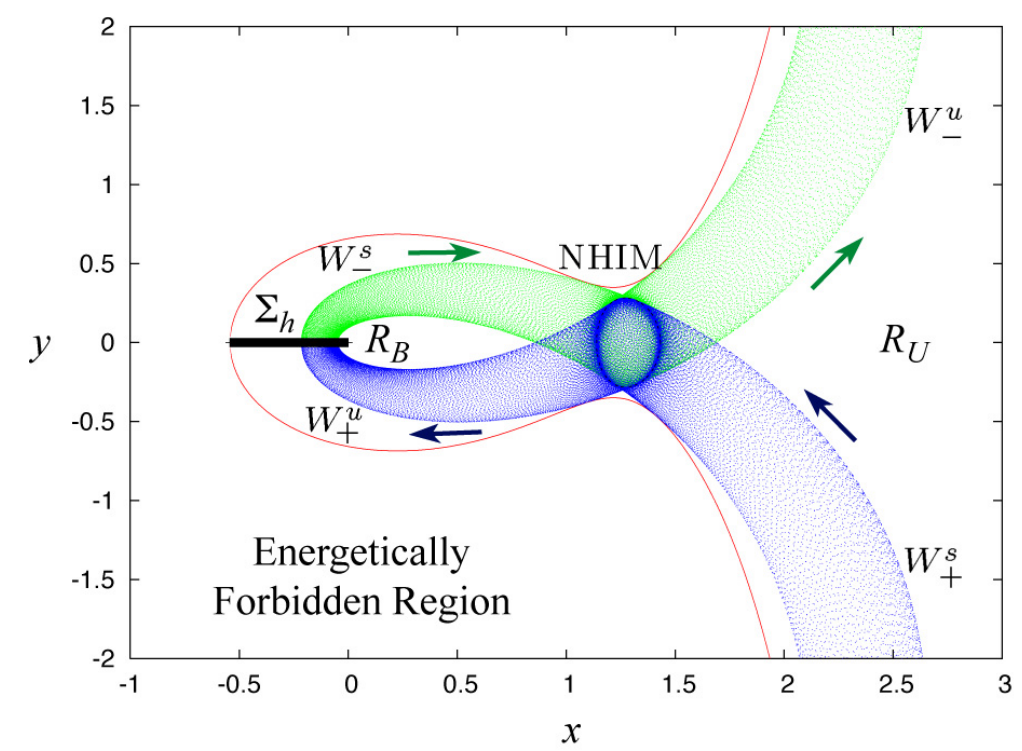

(a)

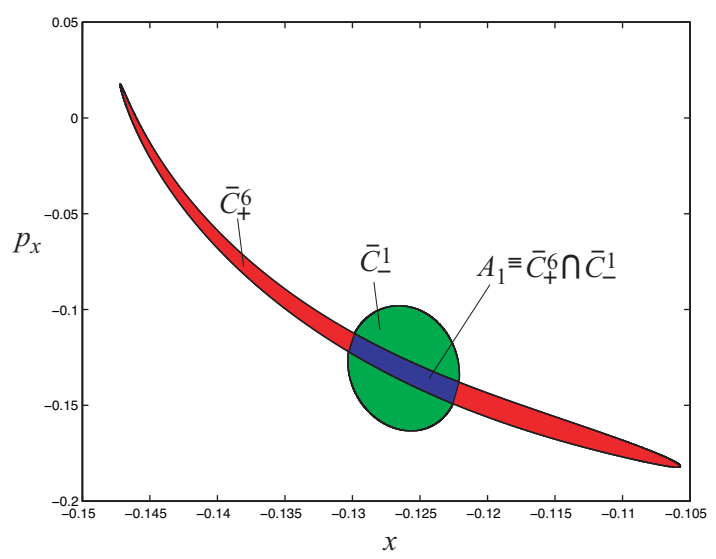

(b)

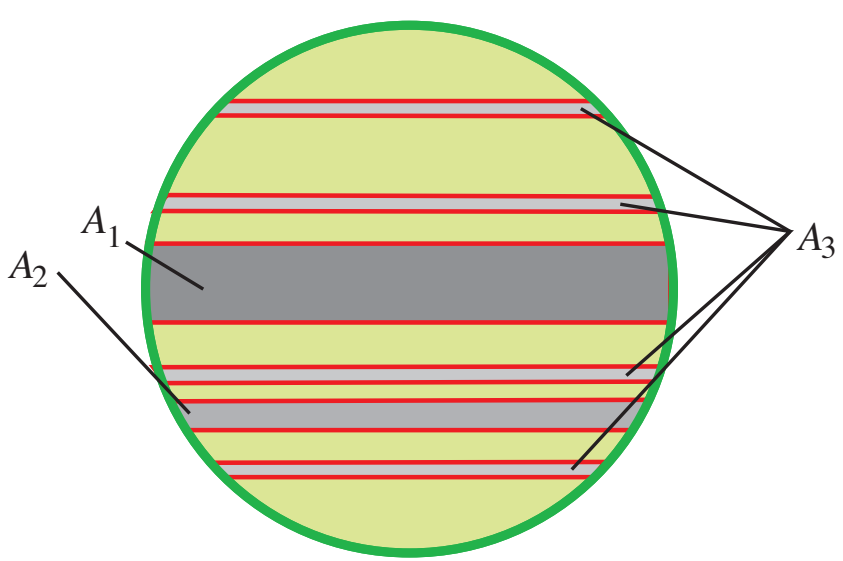

(c)

Figure 4: (a) Stable $\left(W_{ \pm}^{s}\right)$ and unstable $\left(W_{ \pm}^{u}\right)$ tubes of the NHIM in the region $R_{B}$ corresponding to bounded orbits and in the region $R_{U}$ corresponding to unbound orbits. Only planar projections of the tubes are shown. The + branches correspond to incoming reactions from the unbound states to the bound states. The - branches correspond to outgoing reactions from the bound states to the unbound states. $\Sigma_{h}$ denotes the planar projection of the Poincaré section on this energy surface with energy $h$. (b) On $\Sigma_{h}$, the first intersection of the exit $\bar{C}_{-}^{1}$ with an image of the entrance $\bar{C}_{+}^{1}$ is shown. In this case, the smallest $l$ for which $\bar{C}_{+}^{l} \cap \bar{C}_{-}^{1} \neq \emptyset$ is 6 . (c) A schematic of the exit, showing the first intersection, now labeled $A_{1}$, along with subsequent intersections, $A_{2}$ and $A_{3}$. The intersections of successive images of the entrance with the exit, $A_{l}$, will asymptotically cover the entire exit as $l \rightarrow \infty$. 
are what give rise to full-scattering reactions. Moreover, the corresponding intersection volume provide the scattering lifetime distribution and reaction rates. The problem can be simplified by looking only at the intersections of the images of the entrance with the exit itself; that is,

$$
\bar{C}_{+}^{l} \cap \bar{C}_{-}^{1}=f^{l-1}\left(\bar{C}_{+}^{1}\right) \cap \bar{C}_{-}^{1}
$$

where $l=m+k-1$. Figure 4(b) shows the case when $l=6$ for the Rydberg atom in crossed electric and magnetic fields. Any point inside the intersection $\bar{C}_{+}^{6} \cap \bar{C}_{-}^{1}$ is a trajectory that comes from the unbound state $R_{U}$, loops around the bound state region $R_{B}$, and intersects the Poincaré section 6 times before escaping to the unbound state region $R_{U}$. Below, we will use the volume of this kind of intersection to compute the lifetime distribution and reaction rates.

Moreover, the volume enclosed within intersections of successive images of the entrance with the exit will cover the entire exit as $l \rightarrow \infty$. An example of the first intersection is shown in Figure 4(b). The first intersection, labeled $A_{1}$, along with subsequent intersections, $A_{2}$ and $A_{3}$ is shown in Figure 4(c), where schematically we illustrate what occurs for the $A_{l}$ as $l \rightarrow \infty$. Due to the compactness of the bound region chaotic zone in which the tubes meander, the volume enclosed within intersections of successive images of the entrance with the exit will cover the entire exit as $l \rightarrow \infty$. This means that all incoming scattering reactions for which there is a transition from unbound state to bound state will eventually re-react from the bound state to the unbound state as time $t \rightarrow \infty$.

\subsection{Numerical Computation of the Lifetime Distribution Spectrum}

Implementation of the above ideas for full-scattering depends on evaluations of the intersection volumes of the entrance and its images with the exit [6,7, 48].

As we assumed that the dynamics of the system is Hamiltonian, the Poincare map is volume preserving. Let us denote by $V(A)$ the volume of $A \subset \Sigma_{h}$. As we have chosen $\Sigma_{h}$ to be spanned by $(2 n-2)$ conjugate coordinates $\left(q_{2}, p_{2}, \ldots, q_{n}, p_{n}\right)$, we may write

$$
V\left(\bar{C}_{+}^{m}\right)=\int_{\bar{C}_{+}^{m}} d q_{2} d p_{2} \cdots d q_{n} d p_{n} .
$$

Assuming an initially uniform probability distribution of incoming reactants on $\bar{C}_{+}^{1}$, then the fraction of products escaping after executing $m$ loops around the bound region is

$$
\frac{V\left(\bar{C}_{+}^{m} \cap \bar{C}_{-}^{1}\right)}{V\left(\bar{C}_{+}^{1}\right)} .
$$

We will see in Section 3, where we apply this theory to a particular example, that the resulting scattering "spectrum" is structured (that is, it is not a simple exponential decay), and it is closely related to its temporal analogue, i.e., scattering as a function of time. The non-monotonicity of the scattering spectrum has been seen in similar problems in chemistry [7].

Computation of Intersection Volumes via Monte Carlo Methods. To compute the intersection volumes of the Poincaré cuts of the stable and unstable manifolds of the NHIM, we need efficient tools to compute $(2 n-2)$-dimensional volumes, where $n$ is the number of degrees of freedom of the system. For 2 degree of freedom cases, the computation is quite straightforward [35]. However, for higher degrees of freedom, direct computation of volumes with a numerical quadrature is more difficult. The problem of choosing a "good" mesh on the boundary of the $(2 n-2)$-sphere is already very tricky. Hence, a different approach to the computation of these high dimensional volumes is used. We use Monte Carlo methods to compute numerically an approximate value of the $(2 n-2)$-volume. This family of methods are based on a statistical approach to the problem. Thus, they seem to be especially suitable for these kind of situations. 
The basic idea is as follows. We first choose a hyper-rectangle "bounding box" in the $(2 n-2)$ space containing the Poincaré cuts of the stable and unstable manifolds of the NHIM. See Figures 5(a) and 5(b). For the method to be efficient, it is important that this (highdimensional) box contains as tightly as possible the Poincaré cuts. Otherwise, most of the sample points would be "lost" outside the object whose volume we want to compute.

It is then easy to obtain an oracle that distinguishes whether randomly chosen points inside this box belong to the targeted object:

(i) Entrance: A point in $\Sigma_{h}$ belongs to the first Poincaré cut of the capture reaction path $\bar{C}_{+}^{1}$, if the corresponding trajectory has just undergone reaction. This can be checked by numerically integrating the initial condition backward in time, and confirming that the orbit hits some appropriate Poincaré section in the unbound region $R_{U}$.

(ii) Exit: A point in $\Sigma_{h}$ belongs to the first Poincaré cut of the escape reaction path $\bar{C}_{-}^{1}$, if the corresponding trajectory will undergo reaction immediately. This can be checked by numerically integrating the initial condition forward in time, and confirming that the orbit hits some appropriate Poincaré section in the unbound region $R_{U}$.

(iii) mth overlap: A point in $\Sigma_{h}$ belongs to $\bar{C}_{+}^{m} \cap \bar{C}_{-}^{1}$ if it belongs to the exit $\bar{C}_{-}^{1}$ and its $(m-1)$ th backward iterate by the Poincaré map belongs to the entrance (i.e., it belongs to $\left.\bar{C}_{+}^{1}\right)$. This can be checked by showing that the point belongs to the exit, as in (ii), on one hand; and, on the other hand, integrating the initial condition backward in time and confirming that the trajectory hits the Poincaré section an additional $(m-2)$ times before hitting the entrance (checked as in (i)).

Given the oracle, we can use any standard Monte Carlo method to compute the desired volume. In the computations of Section 3, we use importance and stratified sampling in order to reduce the standard deviation and accelerate the convergence of the method [30, 31, 40, 13].

Scattering Profile is Structured and Non-RRKM. In Figures 7(a,c) of Section 3, the percentage of reactants escaping from the bound state as a function of loops in the bound region is shown. The resulting scattering profile, which is derived from the 4D intersection volumes computed via the Monte Carlo integration method, is structured; that is, it is not a simple exponential decay. Moreover, its temporal analogue or lifetime distribution, i.e., scattering as a function of time can also be computed (see Figures $7(b, d)$ ). We note the similarity between the time profile and the "loops" profile. Both results stress that the phase space is not structureless, and that there is a need to take into consideration the tube dynamics and non-RRKM effects when computing reaction rates.

Remarks on Homoclinic and Heteroclinic Intersection Structures. If an intersection on a Poincaré section is between stable and unstable Poincaré cuts related to the same NHIM, it is called homoclinic intersection and if they are related to different NHIMs, heteroclinic intersection. For simplicity, only homoclinic intersection structure has been studied in this paper. But for multi-channel chemical reactions such as isomerization of polyatomic molecules, the study of heteroclinic intersection structures is also needed. Tube dynamics techniques developed in [29] can be very useful for this effort. In our ongoing study of isomerization of triatomic molecules, there are three collinear rank one saddle connecting two triangular isomers.

To study the structure of these intersections, the choice of a suitable set of Poincaré sections will be important. The computation of the volumes of all these different intersections via Monte Carlo methods will be the key step in computing the reaction rates between the two isomers following different reaction channels. 


\section{Application to Rydberg Atom Formation and Ionization}

\subsection{The Hamiltonian Model}

The ionization of a Rydberg atom interacting with external crossed electric and magnetic fields has been studied by other authors (such as $[22,46]$ ). The activation prior to the reaction is given by the initial excitation of a single electron to a high energy level in such a way that its dynamics can be described by classical physics. The reaction takes place when the electron is ionized and detached. Experimentally, an atom is initially prepared in a highly excited Rydberg state and one is interested in its behavior in the future. This is an example of a half-scattering problem.

For the present study, we are instead interested in the full-scattering problem, in which the system is prepared in an unbound initial state and we want to study the dynamics of formation of an excited Rydberg atom and its subsequent ionization. We will use this model problem to illustrate our methodology. The dynamics of the outermost electron in a Rydberg atom in crossed electric and magnetic fields can be described by the following classical Hamiltonian:

$$
H=\frac{1}{2}\left(p_{x}^{2}+p_{y}^{2}+p_{z}^{2}\right)-\frac{1}{r}+\frac{1}{2}\left(x p_{y}-y p_{x}\right)+\frac{1}{8}\left(x^{2}+y^{2}\right)-\varepsilon x,
$$

where $r=\sqrt{x^{2}+y^{2}+z^{2}}$ is the distance from the electron to the center of the nuclear core and $\varepsilon$ is the scaled electric field strength. All the coordinates, as well as the Hamiltonian function, have been scaled by the cyclotron frequency [22].

Using the Legendre transform, one finds that the velocities are given by

$$
\dot{x}=p_{x}-\frac{y}{2}, \quad \dot{y}=p_{y}+\frac{x}{2}, \quad \dot{z}=p_{z} .
$$

The energy in terms of positions and velocities is

$$
E_{\varepsilon}(x, y, z, \dot{x}, \dot{y}, \dot{z})=\frac{1}{2}\left(\dot{x}^{2}+\dot{y}^{2}+\dot{z}^{2}\right)+V_{\varepsilon}(x, y, z),
$$

where the effective potential function is given by

$$
V_{\varepsilon}(x, y, z)=-\frac{1}{r}-\varepsilon x .
$$

The energy integral is the only integral of motion for the system. Notice also that the manifold $z=\dot{z}=0$ is invariant under the dynamics of the full system.

Stark Saddle Point. The vector field of the Rydberg atom has a unique fixed point, which is commonly known as the Stark saddle point:

$$
\begin{aligned}
& x=\frac{1}{\sqrt{\varepsilon}}, \quad y=0, \quad z=0, \\
& \dot{x}=0, \quad \dot{y}=0, \quad \dot{z}=0 .
\end{aligned}
$$

The value of the energy for the Stark point, $E_{S}=-2 \sqrt{\varepsilon}$, is the threshold value for the reaction to take place. This is easily seen by plotting the Hill region in configuration space where the motions of electron is allowed:

$$
\mathcal{H}(\varepsilon, h)=\left\{(x, y, z) \in \mathbb{R}^{3} \mid V_{\varepsilon}(x, y, z) \leq h\right\} .
$$

In Figure 1, the $x y$ planar projection of the Hill region for the three possible cases of the Rydberg atom are shown. Reaction is possible if the energy value of the electron $h$ is higher 


\begin{tabular}{|r|c|c|}
\hline & $\varepsilon=0.58$ & $\varepsilon=0.6$ \\
\hline$\lambda$ & 0.636449792043354 & 0.664862088041162 \\
\hline$\omega_{2}$ & 0.981505729811050 & 0.988576549676131 \\
\hline$\omega_{3}$ & 0.664616310468007 & 0.681731619880499 \\
\hline
\end{tabular}

Table 1: Eigenvalues for the linearized system at the Stark fixed point.

than that of the saddle. i.e., $h>E_{S}$; the critical case is given by the energy value equal to that of the saddle, $h=E_{S}$; and there is no reaction if $h<E_{S}$.

Let $\dot{u}=L u$ be the linearization of the vector field evaluated at the Stark fixed point (see the appendix). Then, the eigenvalues of $L$ describe the linear dynamics around the equilibrium point. For any value of $\varepsilon>0$, we obtain a pair of real eigenvalues $\pm \lambda$ and two pairs of purely imaginary eigenvalues, $\pm i \omega_{2}$ and $\pm i \omega_{3}$ (because of the Hamiltonian character of the vector field, if $\mu$ is an eigenvalue, so are $-\mu, \bar{\mu}$ and $-\bar{\mu})$ :

$$
\pm \lambda= \pm \sqrt{\frac{\sqrt{\alpha^{2}+8 \varepsilon^{3}}-\alpha}{2}}, \quad \pm i \omega_{2}= \pm i \sqrt{\frac{\sqrt{\alpha^{2}+8 \varepsilon^{3}}+\alpha}{2}}, \quad \pm i \omega_{3}= \pm i \varepsilon^{3 / 4},
$$

where $\alpha=1-\varepsilon^{3 / 2}$. Thus, the Stark fixed point is always of the type saddle $\times$ center $\times$ center, and we can call it, indeed, a Stark saddle point. In Table 1, we show the values of $\lambda, \omega_{2}$ and $\omega_{3}$ for $\varepsilon=0.58$ and $\varepsilon=0.6$. Note that values of $\varepsilon>0$ such that

$$
\sqrt{\frac{2 \varepsilon^{3 / 2}}{\sqrt{\alpha^{2}+8 \varepsilon^{3}}+\alpha}} \in \mathbb{Q}
$$

give rise to resonances of the type $k_{2} \omega_{2}-k_{3} \omega_{3}=0$ in the bath modes. However it is important to point out that, in the normal form computations performed in this paper, there are no small divisors. Actually, the denominators appearing in the generating functions (see the appendix) are bounded from below by $|\lambda|$.

\subsection{NHIM and the Stable and Unstable Tubes}

Using the methodology described in the appendix, we construct a high-order normal form of the Hamiltonian near the Stark saddle point (up to order $N=16$ ). This normal form allows us (i) to obtain a very good approximation of the NHIM around the saddle, (ii) to compute the stable and unstable manifold tubes of the NHIM far from the equilibrium point, and (iii) to study their intersections with a well chosen Poincaré section given by

$$
\Sigma_{h, \varepsilon}=\left\{(x, y, z, \dot{x}, \dot{y}, \dot{z}) \in \mathbb{R}^{6} \mid E_{\varepsilon}(x, y, z, \dot{x}, \dot{y}, \dot{z})=h \text { and } y=0, x<0\right\} .
$$

Figure 5 shows the $x \dot{x}$ and $z \dot{z}$ projections of the intersection of the stable and unstable tubes with the Poincaré section $\Sigma_{h, \varepsilon}$ for a particular fixed value of the energy $h=-1.52$ and electric field strength $\varepsilon=0.5835$. In the following numerical experiments, we will fix the energy value to $h=-1.52$ and vary the electric field strength $\varepsilon$. For this level of energy, the Stark saddle point corresponds to an electric field of $\varepsilon_{S}=0.5776$. For $\varepsilon<\varepsilon_{S}$, the bound and unbound regions are disconnected (see Figure 1). For $\varepsilon>\varepsilon_{S}$, the bottleneck at the Stark point is open and becomes wider with larger $\varepsilon$.

The detailed procedure of constructing the high-dimensional tubes and their Poincare cuts is as follows: based on the knowledge of the linear system, we can pick initial conditions which produce a close "shadow" of the stable and unstable manifolds $\left(\simeq S^{3} \times \mathbb{R}\right)$ associated to the 


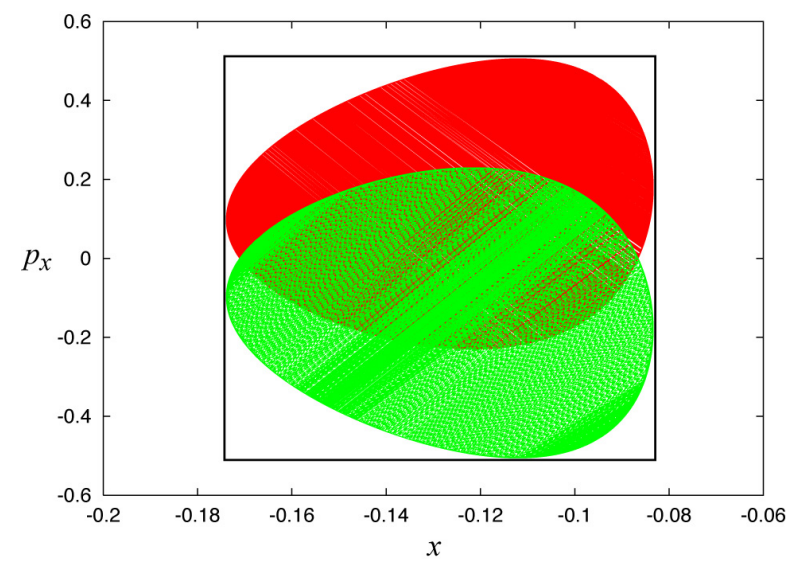

(a)

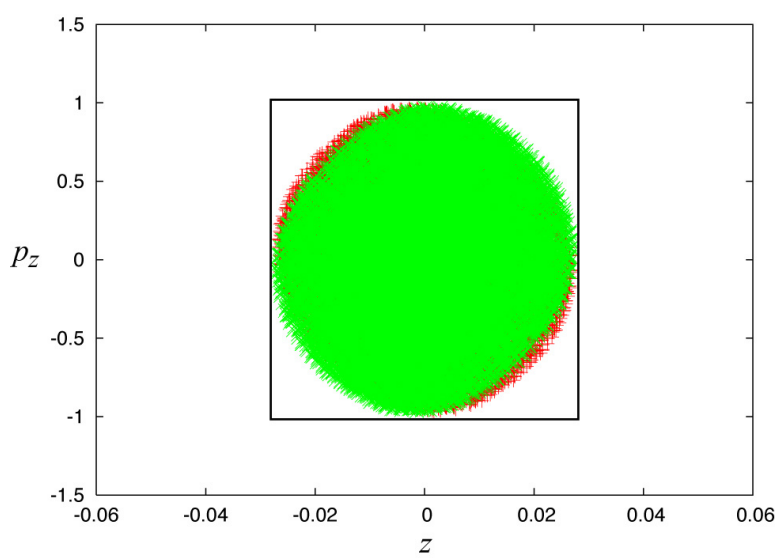

(b)

Figure 5: (a) $x \dot{x}$ and (b) $z \dot{z}$ projections of the intersection of the high-dimensional tubes with the Poincaré section $\Sigma_{h, \varepsilon}$, for $h=-1.52$ and $\varepsilon=0.5835$. The dark sections correspond to the projections of the unstable cuts $\bar{C}_{+}^{1}$ and the light ones to the stable cuts $\bar{C}_{-}^{1}$. The $x \dot{x}$ projection shows partial overlap whereas the $z \dot{z}$ projection shows the cuts nearly completely overlapping.

NHIM. As we restrict to an energy surface with energy $h$, there is only one NHIM per energy surface, denoted $\mathcal{M}_{h}\left(\simeq S^{3}\right)$.

The initial conditions in the normal form coordinates $\left(q_{1}, p_{1}, q_{2}, p_{2}, q_{3}, p_{3}\right)$ are picked with the qualitative picture of the linear system in mind. The coordinates $\left(q_{1}, p_{1}\right)$ correspond to the saddle projection, $\left(q_{2}, p_{2}\right)$ correspond roughly to oscillations within the $(x, y)$ plane, and $\left(q_{3}, p_{3}\right)$ correspond roughly to oscillations within the $z$ direction. Also recall that $q_{3}=p_{3}=0$ $(z=\dot{z}=0)$ corresponds to an invariant manifold of the system, i.e., the planar Rydberg system is an invariant manifold of the three degree of freedom system.

The initial conditions to approximate the stable and unstable manifolds $\left(W_{ \pm}^{s}\left(\mathcal{M}_{h}\right), W_{ \pm}^{u}\left(\mathcal{M}_{h}\right)\right)$ are picked via the following procedure. Note that we can be assured that we are obtaining a roughly complete approximation of points along a slice of $W_{ \pm}^{s}\left(\mathcal{M}_{h}\right)$ and $W_{ \pm}^{u}\left(\mathcal{M}_{h}\right)$ since such a slice is compact, having the structure $S^{3}$. Also, we know roughly the picture from the linear case.

1. We fix $q_{1}=p_{1}= \pm \delta$, where $\delta$ is small. This ensures that almost all of the initial conditions will be for orbits which are transit orbits from one side of the equilibrium region to the other. Specifically + corresponds to right-to-left transit orbits (unbound to bound state reactions) and - corresponds to left-to-right transit orbits (bound to unbound state reactions). We choose $\delta$ small so that the initial conditions are near the NHIM $\mathcal{M}_{h}$ (at $\left.q_{1}=p_{1}=0\right)$ and will therefore integrate forward and backward to be near the unstable and stable manifold of $\mathcal{M}_{h}$, respectively. We choose $\delta$ to not be too small, or the integrated orbits will take too long to leave the vicinity of $\mathcal{M}_{h}$.

2. Beginning with $r_{v}=0$, and increasing incrementally to some maximum $r_{v}=r_{v}^{\max }$, we look for initial conditions with $q_{3}^{2}+p_{3}^{2}=r_{v}^{2}$, i.e. along circles in the $z$ oscillation canonical plane. It is reasonable to look along circles centered on the origin $\left(q_{3}, p_{3}\right)=(0,0)$ on this canonical plane since the motion is simple harmonic in the linear case and the origin corresponds to an invariant manifold.

3. For each point along the circle, we look for the point on the energy surface in the $\left(q_{2}, p_{2}\right)$ plane, i.e., the $(x, y)$ oscillation canonical plane. Note, our procedure can tell us if such a point exists and clearly if no point exists, it will not be used as an initial condition. 
After picking the initial conditions in $\left(q_{1}, p_{1}, q_{2}, p_{2}, q_{3}, p_{3}\right)$ coordinates, we transform to the initial Rydberg coordinates $(x, y, z, \dot{x}, \dot{y}, \dot{z})$ and integrate under the full equations of motion. The integration proceeds until the Poincaré section $\Sigma_{h, \varepsilon}$ stopping condition is reached, in this case $y=0$. We can then use further analysis on the Poincaré section, described below.

Chaotic Sea in the Bounded Region. In the $z=\dot{z}=0$ invariant manifold (which corresponds to the case of planar Rydberg atom), it is possible to visualize the chaotic dynamics of the bounded region $R_{B}$ by plotting the cuts of long-time integration of particular orbits with the Poincare section $\Sigma_{h, \varepsilon}$. For instance, in Figure 6(a), we plot the Poincaré section $y=0$ of the chaotic bounded region for a fixed energy $h=-1.52$ and a scaled electric field strength $\varepsilon=0.57765$, which correspond to values just above the threshold energy of the Stark saddle point. We also show the first five intersections of the stable and unstable tubes of the NHIM with this Poincaré section. In this case, it takes a while until the tubes intersect due to the small aperture of the bottleneck. In Figure 6(b), we show the first 11 intersections of the stable tube with the Poincaré section. Notice the extremely complicated and curling pattern of the manifolds as we iterate forward. 


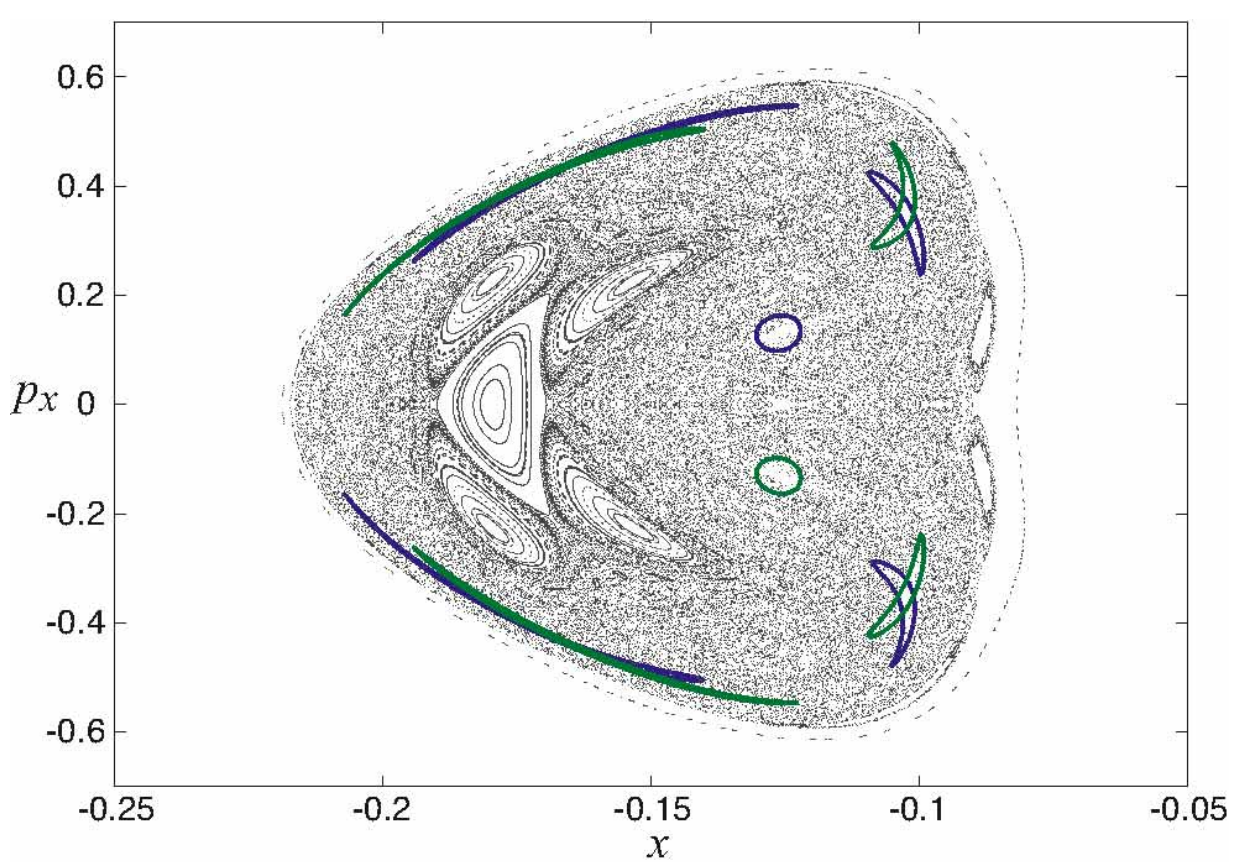

(a)

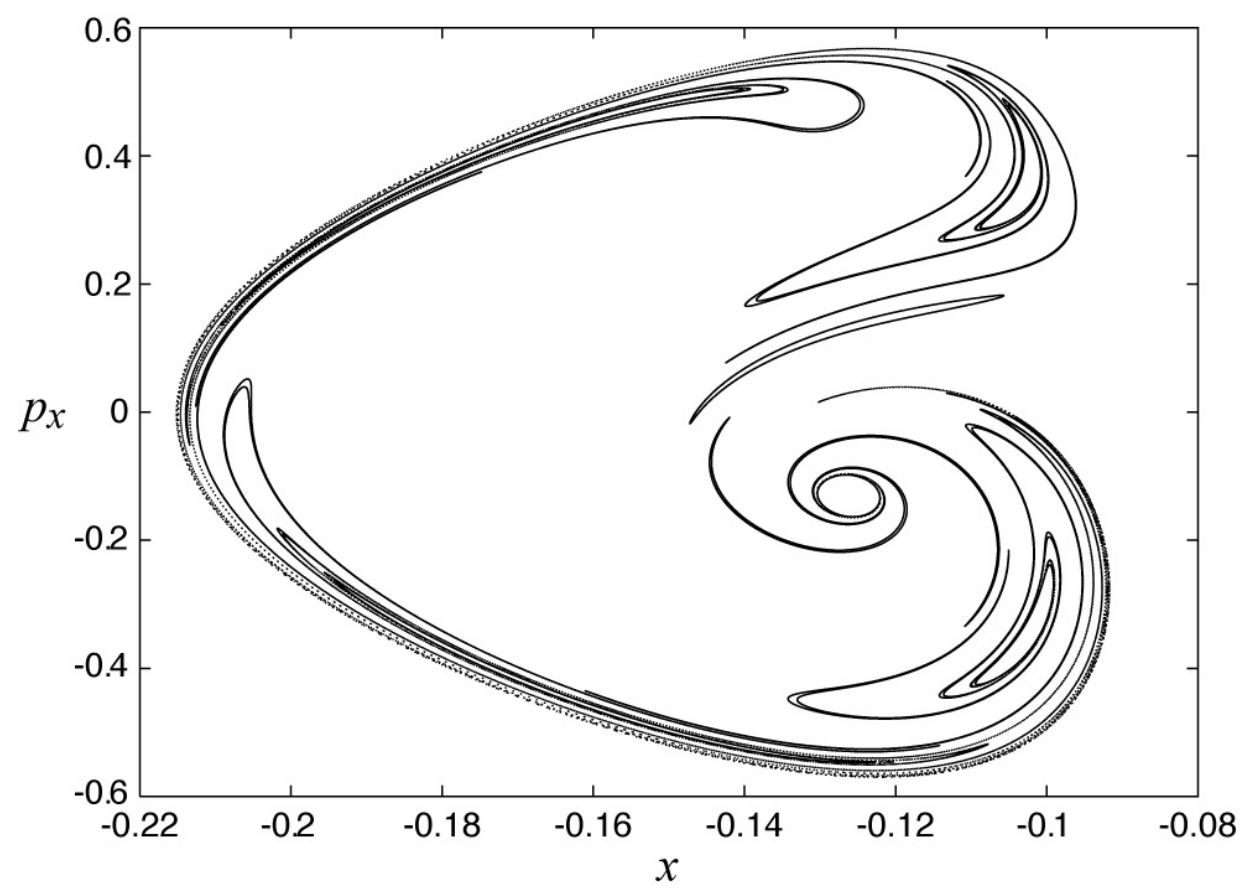

(b)

Figure 6: Poincaré section $\Sigma_{h, \varepsilon}$ in the invariant submanifold $z=\dot{z}=0$. (a) The chaotic sea is shown together with the first five intersections of the tube boundaries and the Poincaré section, $C_{ \pm}^{m}, m=1, \ldots, 5$. A close-up of the intersection of the tubes $\bar{C}_{+}^{6}$ with $\bar{C}_{-}^{1}$ is shown in Figure 4(b). (b) The first eleven intersections of the stable manifold tube boundaries are shown, $C_{-}^{m}, m=1, \ldots, 11$. 


\subsection{Intersection Volumes, Lifetime Distributions, and Rates}

As we explained in Section 2.3, to compute the 4D intersection volumes of the stable and unstable tubes with the Poincaré section we use a Monte Carlo method. First, we choose a hyper-rectangle that contains (as tightly as possible) the intersection volume. This is easily achieved by looking at the 2 projections of the $4 \mathrm{D}$ object on the $x \dot{x}$ and $z \dot{z}$ planes, for instance the ones in Figure 5(a) and (b), respectively. Then, we apply the VEGAS algorithm (see [30,31] and $[40,13])$ to compute the volume of the desired object. Notice, that in order to apply this algorithm we only need a function (or oracle) that tells us whether a given point inside the highdimensional box is contained in the targeted object or not. In this case, this is easily achieved, as explained in Section 2.3: First, we complete the 4-dimensional point to a 6-dimensional phase space initial condition (by imposing the Poincaré section and energy restrictions); and, then, we integrate this point backward in time (to see if it belongs to the entrance, i.e., the green projection in Figure 5), and forward in time (to see if it belongs to the exit, i.e., the dark projection in Figure 5).

Here, we show the results for two examples. First, we consider the case of a fixed energy $h=-1.52$ and scaled electric field strength $\varepsilon=0.58$. The intersection volumes w.r.t. the number of loops of the tube around the nuclear core in the bounded region is shown in Figure 7(a). The scattering spectrum for the $\varepsilon=0.6$ case is shown in Figure $7(\mathrm{c})$.

In Figures 7(b) and 7(d), we show the lifetime distribution of the scattering process. That is, given $N_{i n c}$ incoming electrons coming from the unbound region to form a Rydberg atom, we count how many leave the bound region (ionization of the atom) per unit of time. The practical implementation is as follows: We first generate a quasi-random [37] swarm of points in the box containing the Poincaré cuts by using the Sobol algorithm [44, 1, 40,13]. Then we choose initial conditions such that the corresponding points belong to the entrance $\bar{C}_{+}^{1}$, integrate them forward in time and count how many of them ionize from the atom per unit time. It is clear from the numerical experiments that the resulting lifetime distribution is by no means statistical.

Computation Times. We note that some of the computations done here are parallelizable and future work could take advantage of this to speed up the calculations. All experiments for this work were performed on a PC workstation with an Intel Pentium III 1 Ghz processor. The normal form computations, including the reduction to the center manifold and the construction of the transformation from normal form coordinates to the original coordinates (up to order 16), takes only a number of seconds. The next step, using the normal form to compute 650,000 points on the manifold tube of the NHIM and to numerically integrate them until the first crossing of $\Sigma_{h, \varepsilon}$ (using Runge-Kutta-Fehlberg 7-8) takes about 7 hours. The Monte Carlo computation using $10^{6}$ points takes about two days. Furthermore, we found that using only half as many points for the Monte Carlo portion gave very similar answers. 


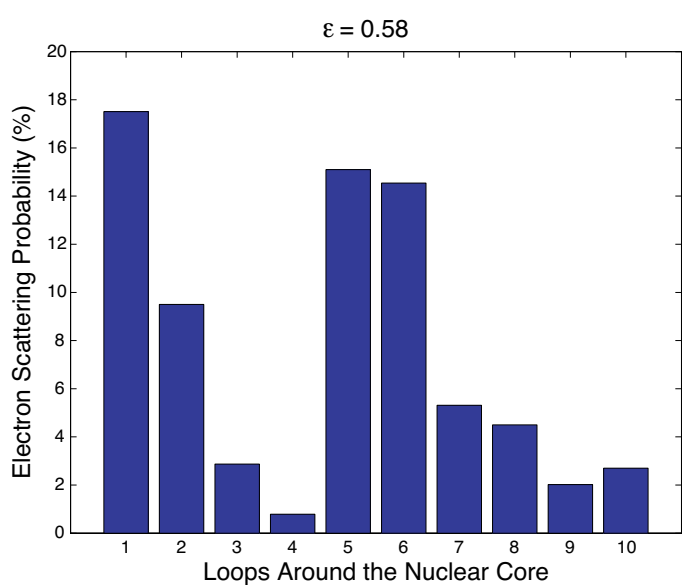

(a)

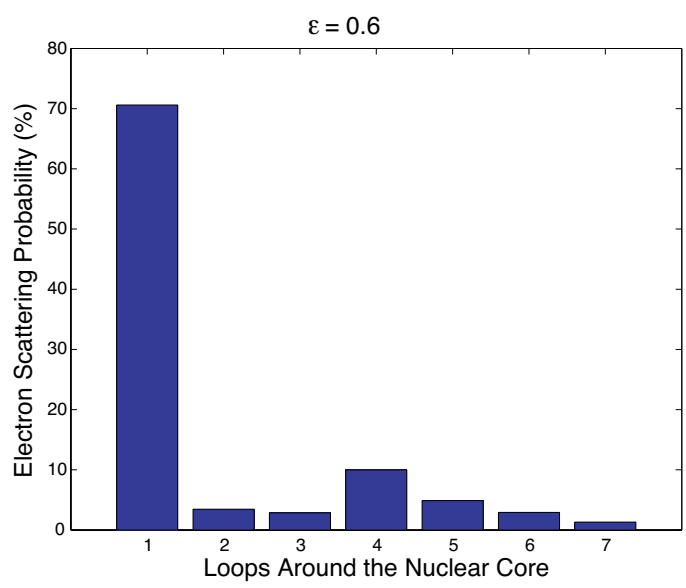

(c)

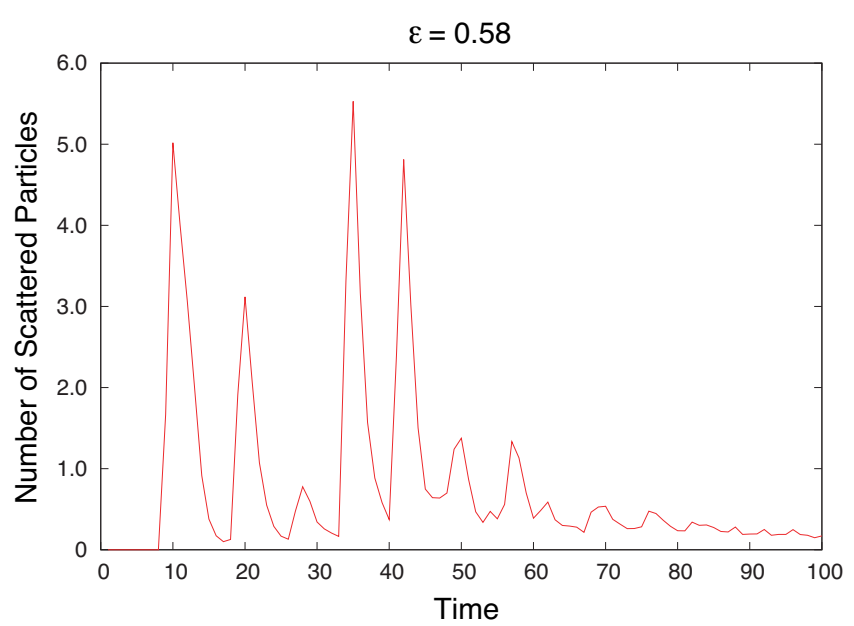

(b)

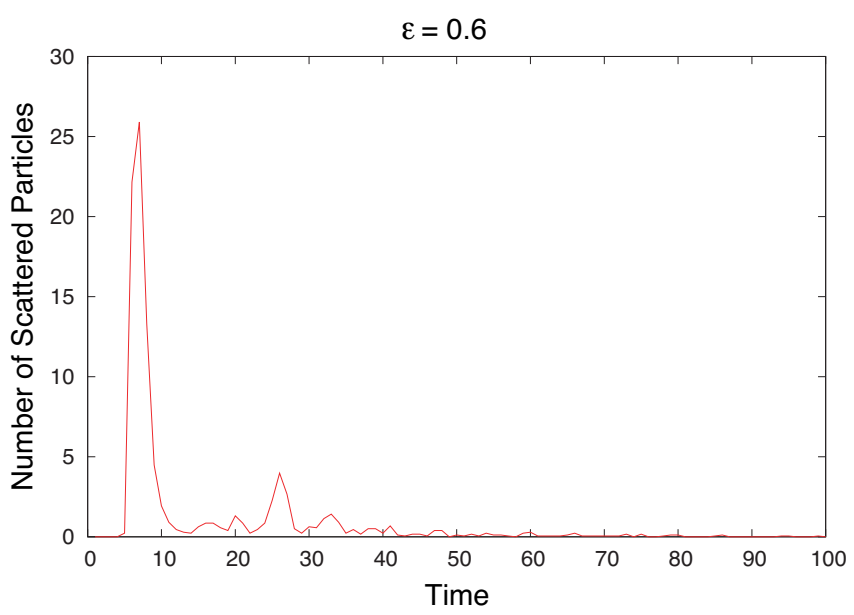

(d)

Figure 7: The electron scattering lifetime distribution for the Rydberg atom in crossed electric and magnetic fields for an energy level $h=-1.52$. (a) The percentage of electrons escaping from the interior region (i.e., scattering away from the bounded region) as a function of loops around the central core is shown in the case $\varepsilon=0.58$. The resulting scattering profile is structured (i.e., not a simple exponential decay), and it is closely related to its temporal analogue, i.e., scattering as a function of time: (b). The non-monotonicity of the scattering spectrum has been seen in similar problems in chemistry and is a hallmark of non-RRKM behavior [5]. (c) and (d): the same for a strength of $\varepsilon=0.6$. For these computations, $10^{6}$ random initial conditions were used. 


\section{Discussion}

This paper has introduced a new method for computing scattering and reaction rates in $n$ degree of freedom systems using tube dynamics in a synergistic way with Monte Carlo volume determination methods. The method was applied to the three degree of freedom model problem of a Rydberg atom. The technique is not restricted by dimension and is useful for systems of more degrees of freedom such as various isomerization problems. This overcomes a major hurdle encountered by De Leon et al. [6,7]. The method can be used in any system with rank-one saddles which separate phase space regions corresponding to different states of the chemical system, such as the isomerization of polyatomic clusters (3 to 7 atoms) $[21,52,53]$ and bimolecular reactions [49].

Our primary concern in this paper is to present and computationally implement a method that is extendable to multidimensional systems. As such, we compute a large number of sample points on the manifolds of the NHIM. However, this is computationally time-consuming, and we expect further refinements will make this part of the process more efficient. This is an area we are currently pursuing. For instance, we might not need to compute a sampling of the entire NHIM manifolds. Since all we need is a bounding box for the Monte Carlo volume computation, we could pick only those points on the NHIM which lead to the largest projections on the Poincaré section. For example, consider Figure 5 . The $x$ and $\dot{x}$ directions of the bounding box could be obtained by looking only at the set on the tube for which $z=\dot{z}=0$.

One could also perhaps obtain the bounding box from the linear dynamics, which will be a good approximation for energies just above the saddle point. Once a bounding box is obtained for small excess energies, numerical continuation could be used to provide a tight bounding box for larger excess energies. This is still work in progress.

Set Oriented Methods. In [8], a completely different approach is taken to compute transport rates in the same Rydberg atom problem. It is based on the Dellnitz-Junge set oriented methods for computing almost invariant sets and transport rates; see [9] and references therein for background. The set oriented approach is also able to deal with both the 2 dimensional and the 3 dimensional problem and gives rates that agree with those given here. Two of the main differences in how the computation is done are as follows. First of all, the tube cross sections are computed not by using normal form methods, but by means of time of return maps and adaptive subdivision techniques; the tubes are then flowed out as in the present paper. Second, the set oriented method, being based on a tree-structured adaptive box subdivision method is able to compute volumes by directly counting the relevant boxes involved, as opposed to using Monte Carlo methods. The fact that the two methods give the same specific results is confirmation that each technique has a solid mathematical and computational foundation.

\section{Acknowledgments}

The authors thank Tomohiro Yanao for interesting discussions and comments; in particular, for suggesting the use of Monte Carlo methods for the calculation of volumes. We thank Charlie Jaffé and Turgay Uzer for originally suggesting the Rydberg atom as an interesting example. We also thank Michael Dellnitz, Oliver Junge, Katalin Grubbits, Kathrin Padberg, and Bianca Thiere for sharing their methods, which make use of set oriented methods applied to the Rydberg atom problem; happily their results, while quite different in methodology, agree with the results obtained here.

This work was partly supported by the California Institute of Technology President's Fund, NSF-ITR grant ACI-0204932 and by ICB, the Institute for Collaborative Biology, through ARO grant DAAD19-03-D-0004. F.G. acknowledges the support of the Fulbright-GenCat post- 
doctoral program, the MCyT/FEDER Grant BFM2003-07521-C02-01 and the CIRIT grant 2001SGR-70. S.D.R. acknowledges the support of National Science Foundation postdoctoral fellowship grant NSF-DMS 0402842.

\section{A Effective Computation of NHIM and its Stable and Un- stable Manifolds}

For the convenience of reader, we have included in this appendix a brief description of the theoretical basis and the practical steps for developing the software used in the numerical explorations of Section 3 (for more details, see [24]).

The Hamiltonian. Recall that the dynamics of the Rydberg atom can be described by the following Hamiltonian function written in normalized coordinates as:

$$
H=\frac{1}{2}\left(p_{x}^{2}+p_{y}^{2}+p_{z}^{2}\right)-\frac{1}{r}+\frac{1}{2}\left(x p_{y}-y p_{x}\right)+\frac{1}{8}\left(x^{2}+y^{2}\right)-\varepsilon x,
$$

where $r=\sqrt{x^{2}+y^{2}+z^{2}}$ is the distance from the electron to the nucleus of the atom and $\varepsilon$ is the scaled electric field.

Linear Behavior and Expansion. First, we compute the linear normal form around the equilibrium point. This is achieved by translating the origin to the fixed point and by using the linearization of the vector field at the origin.

The translation that puts the Stark saddle point at the origin of the phase space is given by

$$
\begin{aligned}
& x=x_{1}+\frac{1}{\sqrt{\varepsilon}}, \quad y=x_{2}, \quad z=x_{3}, \\
& p_{x}=p_{1}, \quad p_{y}=p_{2}-\frac{1}{2 \sqrt{\varepsilon}}, \quad p_{z}=p_{3} .
\end{aligned}
$$

By computing the expansion of the term $\frac{1}{r}$ in Taylor series (a good way of implementing this expansion on a computer is to take advantage of the recurrence of the Legendre polynomials; see for instance, $[24,17])$ :

$$
\frac{1}{r}=\sqrt{\varepsilon}-\varepsilon x_{1}+\varepsilon^{3 / 2} x_{1}^{2}-\frac{1}{2} \varepsilon^{3 / 2}\left(x_{2}^{2}+x_{3}^{2}\right)+\cdots,
$$

and substituting into the translated Hamiltonian, one obtains that its second degree terms (the ones that correspond to the linear dynamics) are

$$
\begin{aligned}
H_{2}= & \frac{1}{2}\left(p_{1}^{2}+p_{2}^{2}+p_{3}^{2}\right)+\frac{1}{2}\left(x_{1} p_{2}-x_{2} p_{1}\right) \\
& +\left(\frac{1}{8}-\varepsilon^{3 / 2}\right) x_{1}^{2}+\left(\frac{1}{8}+\frac{1}{2} \varepsilon^{3 / 2}\right) x_{2}^{2}+\frac{1}{2} \varepsilon^{3 / 2} x_{3}^{2} .
\end{aligned}
$$

This homogeneous polynomial can be put into real normal form by performing a first change of variables consisting in computing the eigenvectors of the linearized vector field at the equilibrium point and using them as the basis of the new reference frame. Then, the quadratic real normal form is

$$
H_{2}=\lambda x p_{x}+\frac{\omega_{2}}{2}\left(y^{2}+p_{y}^{2}\right)+\frac{\omega_{3}}{2}\left(z^{2}+p_{z}^{2}\right) .
$$

Here, $\lambda, \omega_{2}$ and $\omega_{3}$ are positive real numbers given by the linearization of the vector field at the Stark saddle point (see Section 3.1). Note that for simplicity, we have renamed the variables in 
equation (9) as the initial ones $\left(x, y, z, p_{x}, p_{y}, p_{z}\right)$, although they are different after the coordinate change.

For the following normal form computations it is convenient to "diagonalize" the second order terms. This is done by introducing the complex change of coordinates

$$
\left(\begin{array}{c}
y \\
p_{y}
\end{array}\right)=\frac{1}{\sqrt{2}}\left(\begin{array}{cc}
1 & i \\
i & 1
\end{array}\right)\left(\begin{array}{c}
q_{2} \\
p_{2}
\end{array}\right), \quad\left(\begin{array}{c}
z \\
p_{z}
\end{array}\right)=\frac{1}{\sqrt{2}}\left(\begin{array}{cc}
1 & i \\
i & 1
\end{array}\right)\left(\begin{array}{c}
q_{3} \\
p_{3}
\end{array}\right),
$$

where $i=\sqrt{-1}$, and renaming $x=q_{1}$ and $p_{x}=p_{1}$, the second order part of the Hamiltonian becomes

$$
H_{2}=\lambda q_{1} p_{1}+i \omega_{2} q_{2} p_{2}+i \omega_{3} q_{3} p_{3}
$$

From now on we will use the following notation. If $x=\left(x_{1}, \ldots, x_{n}\right)$ is a vector of complex numbers and $k=\left(k_{1}, \ldots, k_{n}\right)$ is an integer vector, we denote by $x^{k}$ the term $x_{1}^{k_{1}} \cdots x_{n}^{k_{n}}$ (in this context we define $0^{0}$ as 1$)$. Moreover, we define $|k|$ as $\sum_{j}\left|k_{j}\right|$.

In order to have all possible orbits in the center manifold, let us expand the initial Hamiltonian $H$ using the coordinates that give us $H_{2}$ as in (11). Then the expanded Hamiltonian takes the form

$$
H(q, p)=H_{2}(q, p)+\sum_{n \geq 3} H_{n}(q, p)=H_{2}(q, p)+\sum_{n \geq 3} h_{i j} q_{1}^{i_{1}} p_{1}^{j_{1}} q_{2}^{i_{2}} p_{2}^{j_{2}} q_{3}^{i_{3}} p_{3}^{j_{3}}
$$

where $H_{2}$ is given in (11) and $H_{n}$ denotes an homogeneous polynomial of degree $n$.

Review of Normal Form Computation. The process of reduction to the center manifold is similar to a normal form computation. The objective is to remove some monomials in the expansion of the Hamiltonian, in order to have an invariant manifold tangent to the center directions of $H_{2}$. For this purpose, let us recall that, if $F(q, p)$ and $G(q, p)$ are two functions of positions, $q$, and momenta, $p$, their Poisson bracket is defined as

$$
\{F, G\}=\sum_{i=0}^{3}\left(\frac{\partial F}{\partial q_{i}} \frac{\partial G}{\partial p_{i}}-\frac{\partial F}{\partial p_{i}} \frac{\partial G}{\partial q_{i}}\right) .
$$

The changes of variables are carried out by means of the Lie series method implemented as in [24] to avoid the Lie triangle with some similarity to [10] (see also [15]). If $G(q, p)$ is a Hamiltonian system, then the function $\hat{H}$ defined by

$$
\hat{H} \equiv H+\{H, G\}+\frac{1}{2 !}\{\{H, G\}, G\}+\frac{1}{3 !}\{\{\{H, G\}, G\}, G\}+\cdots,
$$

is the result of applying a canonical change to $H$. This change is the time one flow corresponding to the Hamiltonian $G$. $G$ is usually called the generating function of the transformation (13). See [16] and references therein for more theoretical details. See [11, 12] for applications to the non-autonomous case. Here we will review only the basics of the procedure.

Note that if $P$ and $Q$ are two homogeneous polynomials of degree $r$ and $s$ respectively, then $\{P, Q\}$ is an homogeneous polynomial of degree $r+s-2$. This means that if $G_{3}$ is an homogeneous polynomial of degree 3 used as a generating function, then the homogeneous polynomials of degree $n, \hat{H}_{n}$, such that $\hat{H}=\sum_{n \geq 2} \hat{H}_{n}$ are given by,

$$
\begin{aligned}
\hat{H}_{2} & =H_{2}, \\
\hat{H}_{3} & =H_{3}+\left\{H_{2}, G_{3}\right\}, \\
\hat{H}_{4} & =H_{4}+\left\{H_{3}, G_{3}\right\}+\frac{1}{2 !}\left\{\left\{H_{2}, G_{3}\right\}, G_{3}\right\} . \\
\ldots & \cdots
\end{aligned}
$$


If we are interested in removing all the terms of order three in the new Hamiltonian, i.e. to have $\hat{H}_{3}=0$, we must choose $G_{3}$ such that it solves the homological equation $\left\{H_{2}, G_{3}\right\}=-H_{3}$.

This procedure can be used recurrently trying to find an homogeneous polynomial of degree four, $G_{4}$, to remove all the terms of order four of the new Hamiltonian, $\hat{H}$, and so on. Nevertheless, we must point out that this is not always possible and some resonant terms, even of order four, cannot be canceled. It is well known that this sequence of Hamiltonians and canonical transformations produced by the Birkhoff normalization does not converge on any open neighborhood of the equilibrium point [39]. Anyway, this process is used to compute what is known as the Birkhoff normal form of the Hamiltonian, having the minimum number of monomials up to some degree.

Reduction to the Center Manifold. Although the reduction to the center manifold is based on this scheme, we only need to remove the instability associated with hyperbolic character of the Hamiltonian $H$. We note that the second order part of the Hamiltonian $\mathrm{H}_{2}$ gives us the linear part of the Hamiltonian equations, and so, the instability is associated with the term $\lambda q_{1} p_{1}$. For this linear approximation of the Hamiltonian equations, the center part can be obtained by setting $q_{1}=p_{1}=0$. If we want the trajectory remains tangent to this space (i.e., having $q_{1}(t)=p_{1}(t)=0$ for all $t>0$ ) when adding the nonlinear terms, we need to have $\dot{q}_{1}(0)=\dot{p}_{1}(0)=0$. Then, because of the autonomous character of the Hamiltonian system, we will obtain $q_{1}(t)=p_{1}(t)=0$ for all $t \geq 0$.

Recalling that the Hamiltonian equations associated with a Hamiltonian $H(q, p)$ are,

$$
\dot{q}_{i}=\frac{\partial H}{\partial p_{i}}, \quad \dot{p}_{i}=-\frac{\partial H}{\partial q_{i}} .
$$

In particular,

$$
\begin{aligned}
& \dot{q}_{1}=\frac{\partial H}{\partial p_{1}}=\lambda q_{1}+\sum_{n \geq 3} h_{i j} q_{1}^{i_{1}} p_{1}^{j_{1}-1} q_{2}^{i_{2}} p_{2}^{j_{2}} q_{3}^{i_{3}} p_{3}^{j_{3}} \\
& \dot{p}_{1}=-\frac{\partial H}{\partial q_{1}}=-\lambda p_{1}-\sum_{n \geq 3} h_{i j} q_{1}^{i_{1}-1} p_{1}^{j_{1}} q_{2}^{i_{2}} p_{2}^{j_{2}} q_{3}^{i_{3}} p_{3}^{j_{3}} .
\end{aligned}
$$

One can get the required condition, $\dot{q}_{1}(0)=\dot{p}_{1}(0)=0$ when $q_{1}(0)=p_{1}(0)=0$, if for instance in the series expansion of the Hamiltonian $H$, all the monomials, $h_{i j} q^{i} p^{j}$, with $i_{1} \neq j_{1}$ have $h_{i j}=0$. This happens, for instance, if there are no monomials with $i_{1} \neq j_{1}$.

More concretely, the actual nonlinear changes of variables are given by the solution of the homological equations. For instance, at order $r>2$, it is not difficult to see that the change given by the following generating function kills the prescribed monomials:

$$
G_{r}(q, p)=\sum_{\substack{i_{1} \neq j_{1} \\|i|+|j|=r}} \frac{-h_{i j}}{\left(j_{1}-i_{1}\right) \lambda+i\left(j_{2}-i_{2}\right) \omega_{2}+i\left(j_{3}-i_{3}\right) \omega_{3}} q^{i} p^{j}
$$

where $q^{i} p^{j}=q_{1}^{i_{1}} p_{1}^{j_{1}} q_{2}^{i_{2}} p_{2}^{j_{2}} q_{3}^{i_{3}} p_{3}^{j_{3}}$. See $[43,25,18]$ for other killing criteria.

All the computations discussed above have been implemented by adapting to our case the software in [24], which uses specific symbolic manipulators in $\mathrm{C}++$ that can do all the procedure up to an arbitrary order. For practical purposes, and in order to have an acceptable equilibrium between precision and time computing requirements, the normal form scheme has been implemented up to order $N=16$.

After all these changes of variables, the initial complexified Hamiltonian around the collinear libration points has been expanded in the following form

$$
H(q, p)=\bar{H}_{N}(q, p)+R_{N}(q, p),
$$


where $\bar{H}_{N}(q, p)$ is a polynomial of degree $N$ without terms of $i_{1} \neq j_{1} . R_{N}(q, p)$ is a remainder of order $N+1$ which is very small near the Stark saddle point and will be skipped in further computations.

Finally, using the inverse change of variables of (10), the truncated Hamiltonian $\bar{H}_{N}$ can be expanded in real form and we obtain

$$
\bar{H}_{N}(q, p)=H_{2}(q, p)+\sum_{n=3}^{N} H_{n}(q, p)
$$

where explicitly,

$$
H_{2}(q, p)=H_{2}\left(q_{1}, p_{1}, q_{2}, p_{2}, q_{3}, p_{3}\right)=\lambda q_{1} p_{1}+\frac{\omega_{2}}{2}\left(q_{2}^{2}+p_{2}^{2}\right)+\frac{\omega_{3}}{2}\left(q_{3}^{2}+p_{3}^{2}\right) .
$$

For convenience, the variables are called again $q, p$.

NHIM and its Stable and Unstable Manifolds. As all the monomials of $H_{n}$ in (14) with $i_{1} \neq j_{1}$ have been eliminated, the truncated Hamiltonian $\bar{H}_{N}$ has a first integral, $I=q_{1} p_{1}$. This is clear since $\bar{H}_{N}$ is given by

$$
\bar{H}_{N}=H_{2}\left(I, q_{2}, p_{2}, q_{3}, p_{3}\right)+\sum_{n=3}^{N} H_{n}\left(I, q_{2}, p_{2}, q_{3}, p_{3}\right) .
$$

Let $f$ be a function of the center manifold variables $\left(q_{2}, p_{2}, q_{3}, p_{3}\right)$ defined as follow

$$
f\left(q_{2}, p_{2}, q_{3}, p_{3}\right)=\sum_{n=3}^{N} H_{n}\left(0, q_{2}, p_{2}, q_{3}, p_{3}\right) .
$$

Then, $f$ is at least of third order. Notice that the invariant manifold $\mathcal{M}_{h}^{3}$ defined by

$$
\mathcal{M}_{h}^{3}=\left\{(q, p) \mid \frac{\omega_{2}}{2}\left(q_{2}^{2}+p_{2}^{2}\right)+\frac{\omega_{3}}{2}\left(q_{3}^{2}+p_{3}^{2}\right)+f\left(q_{2}, p_{2}, q_{3}, p_{3}\right)=h, \quad q_{1}=p_{1}=0\right\}
$$

is the normally hyperbolic invariant manifold (NHIM) for the nonlinear system which corresponds to the 3-sphere (3) for the linearized system. In a small neighborhood of the equilibrium point, since the higher order terms in $f$ are much smaller than the second order terms, the 3 -sphere for the linear problem becomes a deformed sphere for the nonlinear problem. Moreover, since NHIMs persist under perturbation [50], this deformed sphere $\mathcal{M}_{h}^{3}$ still has stable and unstable manifolds which are given by

$$
\begin{aligned}
& W_{ \pm}^{s}\left(\mathcal{M}_{h}^{3}\right)=\left\{(q, p) \mid \frac{\omega_{2}}{2}\left(q_{2}^{2}+p_{2}^{2}\right)+\frac{\omega_{3}}{2}\left(q_{3}^{2}+p_{3}^{2}\right)+f\left(q_{2}, p_{2}, q_{3}, p_{3}\right)=h, \quad q_{1}=0\right\} \\
& W_{ \pm}^{u}\left(\mathcal{M}_{h}^{3}\right)=\left\{(q, p) \mid \frac{\omega_{2}}{2}\left(q_{2}^{2}+p_{2}^{2}\right)+\frac{\omega_{3}}{2}\left(q_{3}^{2}+p_{3}^{2}\right)+f\left(q_{2}, p_{2}, q_{3}, p_{3}\right)=h, \quad p_{1}=0\right\} .
\end{aligned}
$$

Notice the similarity between the formulas above and those for the linearized problem given in equations (4) and (5), especially given the fact that these two coordinate systems are linked by a near-identity transformation.

\section{References}

[1] I. A. Antonov and V. M. Saleev. An effective method for the computation of $\lambda \mathrm{P}_{\tau}$-sequences. Zh. Vychisl. Mat. i Mat. Fiz., 19(1):243-245, 271, 1979. 
[2] D. F. Appleyard. Invariant Sets near the Collinear Lagrangian Points of the Nonlinear Restricted Three-Body Problem. PhD thesis, University of Wisconsin, 1970.

[3] C. C. Conley. Low energy transit orbits in the restricted three-body problem. SIAM J. Appl. Math., 16:732-746, 1968.

[4] C. C. Conley. On the ultimate behavior of orbits with respect to an unstable critical point. I. Oscillating, asymptotic, and capture orbits. J. Differ. Equations, 5:136-158, 1969.

[5] N. De Leon. Cylindrical manifolds and reactive island kinetic theory in the time domain. J. Chem. Phys., 96:285-297, 1992.

[6] N. De Leon, M. A. Mehta, and R. Q. Topper. Cylindrical manifolds in phase space as mediators of chemical reaction dynamics and kinetics. I. Theory. J. Chem. Phys., 94:83108328, 1991.

[7] N. De Leon, M. A. Mehta, and R. Q. Topper. Cylindrical manifolds in phase space as mediators of chemical reaction dynamics and kinetics. II. Numerical considerations and applications to models with two degrees of freedom. J. Chem. Phys., 94:8329-8341, 1991.

[8] M. Dellnitz, K. A. Grubits, J. E. Marsden, K. Padberg, and B. Thiere, Set oriented computation of transport rates in 3-degree of freedom systems: scattering rates for the Rydberg atom in crossed fields, in preparation, 2005.

[9] M. Dellnitz, O. Junge, W. S. Koon, F. Lekien, M. W. Lo, J. E. Marsden, K. Padberg, R. Preis, S. Ross, and B. Thiere, Transport in dynamical astronomy and multibody problems, Intern. J. of Bifurcation and Chaos (to appear), 2005.

[10] A. Deprit. Canonical Transformation Depending on a Small Parameter. Celest. Mech. Dyn. Astr., 1:12-30, 1969.

[11] F. Gabern and À. Jorba. A restricted four-body model for the dynamics near the Lagrangian points of the Sun-Jupiter system. Discrete Contin. Dynam. Systems - Series B, 1(2):143$182,2001$.

[12] F. Gabern and À. Jorba. Effective computation of the dynamics around a two-dimensional torus of a Hamiltonian system. J. Nonlinear Sci. (to appear), 2005.

[13] M. Galassi et al. GNU Scientific Library Reference Manual. GNU Project, second edition, 2004.

[14] R. G. Gilbert and S. C. Smith. Theory of Unimolecular and Recombination Reactions. Blackwell Science Inc, first edition, 1990.

[15] A. Giorgilli. Quantitative methods in classical perturbation theory. In A.E. Roy and B.D. Steves, editors, From Newton to chaos: modern techniques for understanding and coping with chaos in N-body dynamical systems. Plenum Press, New York, 1995.

[16] A. Giorgilli, A. Delshams, E. Fontich, L. Galgani, and C. Simó. Effective stability for a Hamiltonian system near an elliptic equilibrium point, with an application to the restricted three body problem. J. Differ. Equations, 77:167-198, 1989.

[17] G. Gómez, À. Jorba, J. Masdemont, and C. Simó. Dynamics and mission design near libration points. Vol.IV, volume 5 of World Scientific Monograph Series in Mathematics. World Scientific Publishing Co. Inc., River Edge, NJ, 2001. Advanced methods for triangular points.

[18] G. Gómez, W. S. Koon, M. W. Lo, J. E. Marsden, J. Masdemont, and S. D. Ross. Connecting orbits and invariant manifolds in the spatial restricted three-body problem. Nonlinearity, 17(5):1571-1606, 2004. 
[19] A. Haro. Center and center-(un)stable manifolds of elliptic-hyperbolic fixed points of 4Dsymplectic maps. An example: the Froeschlé map. In Hamiltonian systems with three or more degrees of freedom (S'Agaró, 1995), volume 533 of NATO Adv. Sci. Inst. Ser. C Math. Phys. Sci., pages 403-407. Kluwer Acad. Publ., Dordrecht, 1999.

[20] R. J. Hinde and R. S. Berry. Chaotic dynamics in small inert gas clusters: The influence of potential energy saddles. J. Chem. Phys., 99:2942-2963, 1993.

[21] A. Inoue-Ushiyama and K. Takatsuka. Semiclassical quantization of strongly chaotic vibrations in an $M_{7}$-like cluster. Phys. Rev. E, 64:056223, 2001.

[22] C. Jaffé, D. Farrelly, and T. Uzer. Transition state in atomic physics. Phys. Rev. A, 60(5):3833-3850, 1999.

[23] C. Jaffé, S. D. Ross, M. W. Lo, J. Marsden, D. Farrelly, and T. Uzer. Statistical Theory of Asteroid Escape Rates. Phys. Rev. Lett., 89(1):011101, 2002.

[24] À. Jorba. A methodology for the numerical computation of normal forms, centre manifolds and first integrals of Hamiltonian systems. Exp. Math., 8(2):155-195, 1999.

[25] À. Jorba and J. Masdemont. Dynamics in the centre manifold of the collinear points of the Restricted Three Body Problem. Phys. D, 132:189-213, 1999.

[26] T. Komatsuzaki and R. S. Berry. Dynamical hierarchy in transition states: Why and how does a system climb over a mountain? P. Natl. Acad. Sci. USA, 98:7666-7671, 2001.

[27] T. Komatsuzaki and R. S. Berry. Regularity in chaotic reaction paths III: $A r_{6}$ local invariances at the reaction bottleneck. J. Chem. Phys., 115:4105-4117, 2001.

[28] T. Komatsuzaki and R. S. Berry. A Dynamical Propensity Rule for Transitions in Chemical Reactions. J. Phys. Chem. A, 106:10945-10950, 2002.

[29] W. S. Koon, M. W. Lo, J. E. Marsden, and S. D. Ross. Heteroclinic connections between periodic orbits and resonance transitions in celestial mechanics. Chaos, 10(2):427-469, 2000.

[30] G. P. Lepage. A new algorithm for adaptive multidimensional integration. J. Comput. Phys., 27:192-203, 1978.

[31] G. P. Lepage. Vegas: An adaptive multidimensional integration program. Cornell Preprint, CLNS, 1980.

[32] R. A. Marcus. Skiing the Reaction Rate Slopes. Science, 256:1523-1524, 1992.

[33] C. C. Marston and N. De Leon. Reactive islands as essential mediators of unimolecular conformational isomerization: A dynamical study of 3-phospholene. J. Chem. Phys., 91:3392-3404, 1989.

[34] R. McGehee. Some homoclinic orbits for the restricted three-body problem. PhD thesis, University of Wisconsin, Madison, 1969.

[35] J.D. Meiss. Symplectic maps, variational principles, and transport. Rev. Modern Phys., 64(3):795-848, 1992.

[36] J. Moser. On the generalization of a theorem of A. Lyapunov. Comm. Pure Appl. Math., 11:257-271, 1958.

[37] H. Niederreiter. Random number generation and quasi-Monte Carlo methods. Society for Industrial and Applied Mathematics, first edition, 1992.

[38] A. M. Ozorio de Almeida, N. De Leon, M. A. Mehta, and C. C. Marston. Geometry and dynamics of stable and unstable cylinders in Hamiltonian systems. Phys. D, 46(2):265-285, 1990. 
[39] R. Pérez-Marco. Convergence or generic divergence of the Birkhoff normal form. Ann. of Math. (2), 157(2):557-574, 2003.

[40] W. H. Press, S. A. Teukolsky, W. T. Vetterling, and B. P. Flannery. Numerical recipes in C. Cambridge University Press, Cambridge, second edition, 1992. The art of scientific computing.

[41] S. D. Ross. Cylindrical manifolds and tube dynamics in the restricted three-body problem. $\mathrm{PhD}$ thesis, California Institute of Technology, 2004.

[42] C. Simó. On the analytical and numerical approximation of invariant manifolds. In D. Benest and C. Froeschlé, editors, Modern methods in celestial mechanics, pages 285-330. Ed. Frontières, 1990.

[43] C. Simó. Effective computations in Hamiltonian dynamics. In Mécanique céleste: Cent ans après la publication des Méthodes nouvelles de Henri Poincaré, pages 1-23. Soc. Math. France, Paris, 1996.

[44] I. M. Sobol' ${ }^{\prime}$ Distribution of points in a cube and approximate evaluation of integrals. $\breve{Z}$. Vyčisl. Mat. i Mat. Fiz., 7:784-802, 1967.

[45] D. G. Truhlar, B. C. Garrett, and S. J. Klippenstein. Current Status of Transition-State Theory. J. Phys. Chem., 100:12771-12800, 1996.

[46] T. Uzer, C. Jaffé, J. Palacián, P. Yanguas, and S. Wiggins. The geometry of reaction dynamics. Nonlinearity, 15(4):957-992, 2002.

[47] H. Waalkens, A. Burbanks, and S. Wiggins. Phase space conduits for reaction in multidimensional systems: HCN isomerization in three dimensions. J. Chem. Phys., 121:6207$6225,2004$.

[48] H. Wadi and L. Wiesenfeld. Phase space geometry of chaotic reactive scattering: Gateways, windings, and halos. Phys. Rev. E, 55:271-286, 1997.

[49] L. Wiesenfeld, A. Faure, and T. Johann. Rotational transition states: relative equilibrium points in inelastic molecular collisions. J. Phys. B: At. Mol. Opt. Phys., 36:1319-1335, 2003.

[50] S. Wiggins. Normally hyperbolic invariant manifolds in dynamical systems, volume 105 of Applied Mathematical Sciences. Springer-Verlag, New York, 1994. With the assistance of György Haller and Igor Mezić.

[51] S. Wiggins, L. Wiesenfeld, C. Jaffé, and T. Uzer. Impenetrable Barriers in Phase-Space. Phys. Rev. Lett., 86:5478-5481, 2001.

[52] T. Yanao and K. Takatsuka. Collective coordinates and an accompanying metric force in structural isomerization dynamics of molecules. Phys. Rev. A, 68:032714, 2003.

[53] T. Yanao and K. Takatsuka. Kinematic effects associated with molecular frames in structural isomerization dynamics of clusters. J. Chem. Phys., 120:8924-8936, 2004. 